\title{
Improved Accuracy for Alternating-Direction Methods for Parabolic Equations Based on Regular and Mixed Finite Elements
}

\author{
Todd Arbogast*; Chieh-Sen Huang, $\quad$ Song-Ming Yang!
}

November 22, 2005

\begin{abstract}
An efficient modification by Douglas and Kim of the usual alternating directions method reduces the splitting error from $\mathcal{O}\left(k^{2}\right)$ to $\mathcal{O}\left(k^{3}\right)$ in time step $k$. We prove convergence of this modified alternating directions procedure, for the usual non-mixed Galerkin finite element and finite difference cases, under the restriction that $k / h^{2}$ is sufficiently small, where $h$ is the grid spacing. This improves the results of Douglas and Gunn, who require $k / h^{4}$ to be sufficiently small, and Douglas and Kim, who require that the locally one-dimensional operators commute. We propose a similar and efficient modification of alternating directions for mixed finite element methods that reduces the splitting error to $\mathcal{O}\left(k^{3}\right)$, and we prove convergence in the noncommuting case, provided that $k / h^{2}$ is sufficiently small. Numerical computations illustrating the mixed finite element results are also presented. They show that our proposed modification can lead to a significant reduction in the alternating direction splitting error.
\end{abstract}

\section{Introduction.}

We consider in this paper the approximation of a parabolic problem on a bounded domain $\Omega \subset \mathbb{R}^{d}$ of the form

$$
\begin{aligned}
& c u_{t}-\nabla \cdot(a \nabla u)=f, \quad x \in \Omega, \quad 0<t \leq T, \\
& u=g, \quad x \in \partial \Omega, \quad 0<t \leq T, \\
& u=u_{0}, \quad x \in \Omega, \quad t=0,
\end{aligned}
$$

where $u_{t}$ is the time partial derivative of $u(x, t), c(x)$ and $a(x)$ are uniformly positive on $\bar{\Omega}$, and $c(x), a(x), f(x, t), g(x, t)$, and $u_{0}(x)$ are sufficiently smooth for our purposes. Since the 1950's, scientists have formulated time-stepping procedures to numerically approximate the

\footnotetext{
${ }^{*}$ Department of Mathematics, The University of Texas at Austin, 1 University Station C1200, Austin, Texas 78712, U.S.A.

${ }^{\dagger}$ Department of Applied Mathematics and National Center for Theoretical Sciences, National Sun Yat-sen University, Kaohsiung 804, Taiwan, R.O.C.

${ }^{\ddagger}$ Corresponding author (huangcs@math.nsysu.edu.tw).
} 
solutions of such problems. The Alternating Direction (AD) methods were first introduced in 1955 by Douglas, Peaceman, and Rachford [4, 8, 13]. They noted that

$$
-\nabla \cdot(a \nabla)=-\sum_{i=1}^{d} \frac{\partial}{\partial x_{i}}\left(a \frac{\partial}{\partial x_{i}}\right)
$$

is a sum of $d$ one-dimensional operators and, thereby, treated the spatial variables of (1.1) individually in a cyclic fashion. This locally one-dimensional approach produces a splitting error over an approach that treats the full $d$-dimensional problem at once.

An AD method can be interpreted as being a perturbation of some underlying implicit numerical time-stepping method, such as Crank-Nicolson or backward Euler. The spatial variable splitting error terms form a perturbation of the same order in the time step $k$ as the truncation error terms associated with the Crank-Nicolson method, $\mathcal{O}\left(k^{2}\right)$, and of higher order with the backward Euler method, $\mathcal{O}(k)$. Thus, the asymptotic rate of convergence for the AD method should be of the same order in the spatial and temporal discretization parameters as that for its associated underlying method. However, at practical levels of discretization, the actual errors associated with an $\mathrm{AD}$ method can be much larger than that for the underlying method. To rectify this, Douglas and Kim [7] (cf. [5]) proposed the Modified Alternating Direction iteration algorithms (AD-M), sometimes referred to as AD-II, AD with Improved Initialization (see (2.11)-(2.13) below). They modify the right hand side of an $\mathrm{AD}$ algorithm to reduce the order of the splitting error from $\mathcal{O}\left(k^{2}\right)$ to $\mathcal{O}\left(k^{3}\right)$.

This paper has two main results. In [7], Douglas and Kim give a convergence proof for AD$\mathrm{M}$ under the assumption that the order in which the individual spatial variables are treated is immaterial. More precisely, if $A_{m}$ is the discrete approximation to the one-dimensional operator $-\partial / \partial x_{m}\left(a \partial / \partial x_{m}\right)$, they require that $A_{m_{1}}$ and $A_{m_{2}}$ commute for all $m_{1}$ and $m_{2}$. This condition generally does not hold in practice, for example, when $a$ is not constant. Some 40 years ago, Douglas and Gunn [6] provided a general formulation and proof of convergence of the $\mathrm{AD}$ method in the non-commutative case. However, they required the restriction that $k / h^{4}$ must be sufficiently small, where $h$ is the grid spacing. Our first main result is a convergence proof for the AD-M under the constraint that merely $k / h^{2}$ is sufficiently small.

Our second main result is an improved treatment of mixed finite element methods $[14,3]$. These methods approximate the flux variable $\mathbf{q}=-a \nabla u$ simultaneously with the scalar variable $u$, and they are often employed to solve for flow fields in physics and engineering problems. Douglas and Pietra [9] formulated an AD iterative technique for solving the algebraic systems associated with mixed finite element methods for second order elliptic problems. We propose a modification similar to the AD-M method for the non-mixed formulation. Our modification reduces the splitting error from $\mathcal{O}\left(k^{2}\right)$ to $\mathcal{O}\left(k^{3}\right)$. A similar convergence theory to that our first main result is developed for this mixed AD-M method. That is, we obtain convergence for the noncommuting case with the restriction that $k / h^{2}$ is sufficiently small.

The rest of the paper is organized as follows. In the next section, we define the non-mixed $\mathrm{AD}$ and $\mathrm{AD}-\mathrm{M}$ methods for the usual Galerkin finite element or finite difference discretizations of parabolic equations. We present our convergence proof for AD-M in $\S 3$. After a review of the standard mixed finite element method, given in $\S 4$, the AD-M for mixed finite element methods is formulated in $\S 5$. Our convergence results for mixed methods are given in $\S 6$. Finally, in $\S 7$, we present some numerical experiments illustrating the utility of our mixed methods, and confirming our theoretical results. 


\section{The basic methods in non-mixed form.}

Although our results do not require the following constraint, for simplicity we tacitly suppose that $\Omega \subset \mathbb{R}^{d}$ admits a rectangular spatial grid of maximal spacing $h$. This is the usual situation considered since AD can be implemented efficiently in this case. Also for simplicity, we take a uniform time step $k=T / N>0$. We define discrete times $t^{n}=n k$ and use the notation $\varphi^{n}$ in place of $\varphi\left(t^{n}\right)$ and $\varphi^{n+1 / 2}$ in place of $\varphi\left(\left(t^{n}+t^{n+1}\right) / 2\right)$.

Loosely speaking, we let $A$ be the $d$-dimensional linear operator obtained from finite difference or finite element approximation of $-\nabla \cdot(a \nabla)$ over the grid on $\Omega$ with order of accuracy $\mathcal{O}\left(h^{s}\right)$. We assume that

$$
A=\sum_{m=1}^{d} A_{m},
$$

where each $A_{m}$ can be inverted relatively easily. Normally, $A_{m}$ is a one-dimensional linear operator obtained from approximation of $-\partial_{m}\left(a \partial_{m}\right)$, where $\partial_{m}=\partial / \partial x_{m}$, on an $x_{m}$-line of Omega over the grid. However, we need to be more precise about exactly what we mean by the operator $A_{m}$, especially for the next section where we need to apply it multiple times to the true solution. It is confusing to explain the finite difference and finite element cases together, so we present only the more difficult finite element case and leave it to the reader to translate things to the finite difference case.

We begin by rewriting our differential system $(1.1)-(1.2)$ in variational form. Let $(\cdot, \cdot)$ denote the inner-product in $L^{2}(\Omega)$. We find $u \in H_{0}^{1}(\Omega)+g$, where $g$ is extended to all of $\Omega$, such that

$$
\left(c u_{t}, v\right)+(a \nabla u, \nabla v)=(f, v), \quad v \in H_{0}^{1}(\Omega) .
$$

Let $V_{h} \subset H_{0}^{1}(\Omega)$ denote our finite element space, with nodal basis

$$
V_{h}=\operatorname{span}_{i}\left\{v_{i}\right\}
$$

We will approximate $u$ in $V_{h}+g$. Now $A$ and $A_{m}$ are symmetric, positive semidefinite matrices, with $i j$-entries

$$
A_{i j}=\left(a \nabla v_{i}, \nabla v_{j}\right) \quad \text { and } \quad A_{m, i j}=\left(a \partial_{m} v_{i}, \partial_{m} v_{j}\right)
$$

We also need the positive definite matrix

$$
C_{i j}=\left(c v_{i}, v_{j}\right)
$$

(which is diagonal is mass lumping is used) and the vector $F$ given by

$$
F_{i}=\left(f, v_{i}\right)-\left(c \frac{g^{n+1}-g^{n}}{k}, v_{i}\right)-\left(a \nabla\left[\theta g^{n+1}+(1-\theta) g^{n}\right], \nabla v_{i}\right),
$$

for $\theta$ chosen immediately below. 


\subsection{The backward Euler and Crank-Nicolson methods.}

The basic time-stepping algorithms for (1.1) can be written together using $\theta=1$ for backward Euler and $\theta=1 / 2$ for Crank-Nicolson.

We approximate $u^{n}$ by $u_{h}^{n} \in V_{h}+g^{n}$, which has the expansion

$$
u_{h}^{n}=\sum_{i} U_{i}^{n} v_{i}+g^{n}
$$

in terms of the vector $U^{n}$ which satisfies

$$
C \frac{U^{n+1}-U^{n}}{k}+A\left(\theta U^{n+1}+(1-\theta) U^{n}\right)=F^{n+\theta}, \quad n=0,1, \ldots, N-1,
$$

where $U^{0}$ is the vector of nodal values for some given and accurate approximation to $u_{0}-g^{0}$ in $V_{h}$. It is well known that the local time truncation error is $\mathcal{O}(k)$ for backward Euler and $\mathcal{O}\left(k^{2}\right)$ for Crank-Nicolson, which we can write in the compact form $\mathcal{O}\left(k^{3-2 \theta}\right)$. Unfortunately, we must solve an implicit multi-dimensional problem for each time step.

\subsection{The AD method.}

The Douglas-Gunn algorithm [6] for AD time discretization of (2.3) asks that, for each $n=0,1, \ldots, N-1$, we find $w^{n, m}$, for $m=1, \ldots, d$, such that

$$
C \frac{w^{n, m}-w^{n}}{k}+\sum_{i=1}^{m} A_{i}\left(\theta w^{n, i}+(1-\theta) w^{n}\right)+\sum_{i=m+1}^{d} A_{i} w^{n}=F^{n+\theta},
$$

and then set $w^{n+1}=w^{n, d}$, where $w^{n}$ approximates $u^{n}$ in the usual sense:

$$
w_{\mathrm{AD}, h}^{n}=\sum_{i} w_{i}^{n} v_{i}+g^{n} .
$$

Note that we need to solve implicitly only a one-dimensional problem at each stage. This algorithm is written in the efficiently computable, equivalent form

$$
\begin{aligned}
\left(C+\theta k A_{1}\right) w^{n, 1} & =\left(C-(1-\theta) k A_{1}-k \sum_{i=2}^{d} A_{i}\right) w^{n}+k F^{n+\theta}, \\
\left(C+\theta k A_{m}\right) w^{n, m} & =C w^{n, m-1}+\theta k A_{m} w^{n}, \quad m=2, \ldots, d, \\
w^{n+1} & =w^{n, d} .
\end{aligned}
$$

Multiply (2.7) by $\left(1+\theta k C^{-1} A_{1}\right) \cdots\left(1+\theta k C^{-1} A_{m-1}\right)$, sum on $m$, and add (2.6) to see that

$$
C \frac{w^{n+1}-w^{n}}{k}+A\left(\theta w^{n+1}+(1-\theta) w^{n}\right)+B\left(w^{n+1}-w^{n}\right)=F^{n+\theta},
$$

where

$$
\begin{aligned}
B= & k^{-1} C\left[\left(1+\theta k C^{-1} A_{1}\right) \cdots\left(1+\theta k C^{-1} A_{d}\right)-1-\theta k C^{-1} A\right] \\
= & \theta^{2} k \sum_{1 \leq m_{1}<m_{2} \leq d} A_{m_{1}} C^{-1} A_{m_{2}}+\theta^{3} k^{2} \sum_{1 \leq m_{1}<m_{2}<m_{3} \leq d} A_{m_{1}} C^{-1} A_{m_{2}} C^{-1} A_{m_{3}} \\
& +\cdots+\theta^{d} k^{d-1} A_{1} C^{-1} A_{2} \cdots C^{-1} A_{d} .
\end{aligned}
$$


This equation is similar to (2.3), and from it we conclude, without being very precise, that the splitting error is

$$
B\left(w^{n+1}-w^{n}\right)=k B\left(\frac{w^{n+1}-w^{n}}{k}\right)=\mathcal{O}\left(k^{2} \sum_{i, j}\left|\frac{\partial}{\partial t} \frac{\partial^{2}}{\partial x_{i}^{2}} \frac{\partial^{2}}{\partial x_{j}^{2}}(u-g)\right|\right)=\mathcal{O}\left(k^{2}\right),
$$

provided that $w$ (i.e., $w_{\mathrm{AD}, h}-g$ ) is a good approximation of $u-g$, and $a, c, g$, and $u$ are sufficiently smooth.

\subsection{The AD-M method.}

The Douglas-Kim AD-M algorithm [7] adds a term to the right-hand side of (2.6); that is, for each $n=1,2, \ldots, N-1$, we find $W^{n, m}$ for $m=1, \ldots, d$ such that

$$
\begin{aligned}
\left(C+\theta k A_{1}\right) W^{n, 1}= & \left(C-(1-\theta) k A_{1}-k \sum_{i=2}^{d} A_{i}\right) W^{n} \\
& +k F^{n+\theta}+B\left(W^{n}-W^{n-1}\right), \\
\left(C+\theta k A_{m}\right) W^{n, m}= & C W^{n, m-1}+\theta k A_{m} W^{n}, \quad m=2, \ldots, d, \\
W^{n+1}= & W^{n, d}
\end{aligned}
$$

where $W^{n}$ approximates $u^{n}$ via

$$
w_{h}^{n}=\sum_{i} W_{i}^{n} v_{i}+g^{n}
$$

We retain the property that only one-dimensional problems need to be solved implicitly at each stage. These equations imply

$$
C \frac{W^{n+1}-W^{n}}{k}+A\left(\theta W^{n+1}+(1-\theta) W^{n}\right)+B\left(W^{n+1}-2 W^{n}+W^{n-1}\right)=F^{n+\theta},
$$

from which we see that the splitting error

$$
\begin{aligned}
B\left(W^{n+1}-2 W^{n}+W^{n-1}\right) & =k^{2} B\left(\frac{W^{n+1}-2 W^{n}+W^{n-1}}{k^{2}}\right) \\
& =\mathcal{O}\left(k^{3} \sum_{i, j}\left|\frac{\partial^{2}}{\partial t^{2}} \frac{\partial^{2}}{\partial x_{i}^{2}} \frac{\partial^{2}}{\partial x_{j}^{2}}(u-g)\right|\right)=\mathcal{O}\left(k^{3}\right)
\end{aligned}
$$

is improved in convergence order from the $\mathrm{AD}$ method. The price we pay is that we need some good approximation to $W^{1}$, which we might obtain, for example, by solving AD several times with a smaller time step.

\section{A new proof of convergence for AD-M.}

As noted in the Introduction, Douglas and Kim [7] gave a convergence proof for AD-M under the assumption that the $A_{m}$ commute. Douglas and Gunn [6] handled non-commutative 
problems, but required the restriction that $k h^{-4}$ be sufficiently small. We significantly relax this constraint in this section.

Recall that $(\cdot, \cdot)$ is the inner-product in $L^{2}(\Omega)$, and let $\|\cdot\|$ denote the induced $L^{2}(\Omega)$ norm. We will also use some of the $H^{\ell}(\Omega)$-norms, denoted by $\|\cdot\|_{\ell}$. We make the following reasonable assumptions regarding the underlying discretizations.

Assumption 3.1 For some constant $\mathcal{C}$ independent of $h$ and $k, k h^{-2} \leq \mathcal{C}$. Moreover, for any vector $\Psi \in \mathbb{R}^{\operatorname{dim} V_{h}}$,

$$
|\Psi| \leq \mathcal{C}\|\psi\|
$$

where $\psi=\sum_{i} \Psi_{i} v_{i}$

The latter inequality above holds because the mesh is quasi-uniform.

Assumption 3.2 For some constant $\mathcal{C}$ depending on the smoothness of $a, c$, and $g$, but not on $h$ or $k$, the discrete spatial operators $A_{m}$, and $B$ satisfy:

1. Each $A_{m}$ is bounded in the sense that $\left\|A_{m}\right\| \leq \mathcal{C} h^{-2}$;

2. $B$ satisfies the bound $|B v| \leq \mathcal{C} k h^{-4}|v|$.

Moreover, $0<a_{*} \leq a \leq a^{*}<\infty$ and $0<c_{*} \leq c \leq c^{*}<\infty$ on $\bar{\Omega}$.

Note that Part 1 holds for the usual finite element spaces, and it (with Assumption 3.1) implies Part 2.

Assumption 3.3 For some constant $\mathcal{C}$ depending on the smoothness of $u, a, c$, and $g$, but not on $h$ or $k$, the following hold.

1. The initial approximation is the elliptic projection, meaning that $u_{h}^{0}=w_{h}^{0} \in V_{h}+g^{0}$ satisfies

$$
\left(a \nabla\left(u-u_{h}^{0}\right), \nabla v\right)=0, \quad v \in V_{h} .
$$

Moreover,

$$
\left\|u^{0}-u_{h}^{0}\right\|+h\left\|u^{0}-u_{h}^{0}\right\|_{1} \leq \mathcal{C} h^{s} .
$$

2. The solution $u_{h}$ to the full scheme (2.3) approximates the true solution $u$ of (1.1)-(1.3) in the sense that the error $\mathcal{E}^{n}=u^{n}-u_{h}^{n}$ satisfies

$$
\begin{aligned}
& \left(\sum_{n=0}^{N-1}\left\|\frac{\mathcal{E}^{n+1}-\mathcal{E}^{n}}{k}\right\|^{2} k\right)^{1 / 2}+\max _{0 \leq m \leq N}\left\|\mathcal{E}^{m}\right\| \leq \mathcal{C}\left\{h^{s}+k^{3-2 \theta}\right\}, \\
& \max _{0 \leq m \leq N}\left\|\mathcal{E}^{m}\right\|_{1} \leq \mathcal{C}\left\{h^{s-1}+k^{3-2 \theta}\right\} .
\end{aligned}
$$

It is well known that this assumption holds for the usual $s$-order accurate finite element spaces, when we carefully choose the approximation to the initial condition $[15,1]$. In fact, Part 2 can be proven to hold using techniques similar to what we use in the remainder of this section. 
To prove convergence of AD-M, let $E^{n}=U^{n}-W^{n}$ and subtract (2.15) from (2.3) to obtain

$$
C \frac{E^{n+1}-E^{n}}{k}+A\left(\theta E^{n+1}+(1-\theta) E^{n}\right)=B\left(W^{n+1}-2 W^{n}+W^{n-1}\right) .
$$

In integral form, with $e^{n}=u_{h}^{n}-w_{h}^{n}$, the $i$ th component is

$$
\left(c \frac{e^{n+1}-e^{n}}{k}, v_{i}\right)+\left(a \nabla\left[\theta e^{n+1}+(1-\theta) e^{n}\right], \nabla v_{i}\right)=\left(B\left(W^{n+1}-2 W^{n}+W^{n-1}\right)\right)_{i} .
$$

Take the dot product of this with the test vector $E^{n+1}-E^{n}$, i.e., the test function $e^{n+1}-e^{n}$, to obtain for $n=1,2, \ldots, N-1$,

$$
\begin{aligned}
& \left\|c^{1 / 2} \frac{e^{n+1}-e^{n}}{k}\right\|^{2} k+\theta\left(a \nabla e^{n+1}, \nabla e^{n+1}\right)+(1-2 \theta)\left(a \nabla e^{n+1}, \nabla e^{n}\right)-(1-\theta)\left(a \nabla e^{n}, \nabla e^{n}\right) \\
& =B\left(W^{n+1}-2 W^{n}+W^{n-1}\right) \cdot\left(E^{n+1}-E^{n}\right) .
\end{aligned}
$$

Thus, for either $\theta=1$ or $\theta=1 / 2$,

$$
\begin{aligned}
& c_{*}\left\|\frac{e^{n+1}-e^{n}}{k}\right\|^{2} k+\frac{1}{2}\left(\left(a \nabla e^{n+1}, \nabla e^{n+1}\right)-\left(a \nabla e^{n}, \nabla e^{n}\right)\right) \\
& \quad \leq \mathcal{C}\left|B\left(W^{n+1}-2 W^{n}+W^{n-1}\right)\right|\left\|e^{n+1}-e^{n}\right\| \\
& \quad \leq \mathcal{C}\left|B\left(W^{n+1}-2 W^{n}+W^{n-1}\right)\right|^{2} k+\frac{c_{*}}{2}\left\|\frac{e^{n+1}-e^{n}}{k}\right\|^{2} k .
\end{aligned}
$$

Now for $\Psi \in \mathbb{R}^{\operatorname{dim} V_{h}}$, with $\psi=\sum_{i} \Psi_{i} v_{i}$, note that

$$
\begin{aligned}
B \Psi= & \theta^{2} k \sum_{1 \leq m_{1}<m_{2} \leq d} A_{m_{1}} C^{-1} A_{m_{2}} \Psi+\cdots+\theta^{d} k^{d-1} A_{1} C^{-1} A_{2} \cdots C^{-1} A_{d} \Psi \\
= & \theta^{2} k \sum_{1 \leq m_{1}<m_{2} \leq d} A_{m_{1}} C^{-1}\left(\left(a \partial_{m_{2}} v_{i}, \partial_{m_{2}} \psi\right)\right)_{i} \\
& +\cdots+\theta^{d} k^{d-1} A_{1} C^{-1} A_{2} \cdots C^{-1}\left(\left(a \partial_{d} v_{i}, \partial_{d} \psi\right)\right)_{i} \\
= & \theta^{2} k \sum_{1 \leq m_{1}<m_{2} \leq d} A_{m_{1}} C^{-1}\left(\left(v_{i},-\partial_{m_{2}} a \partial_{m_{2}} \psi\right)\right)_{i} \\
& +\cdots+\theta^{d} k^{d-1} A_{1} C^{-1} A_{2} \cdots C^{-1}\left(\left(v_{i},-\partial_{d} a \partial_{d} \psi\right)\right)_{i} .
\end{aligned}
$$

In this way, we see that $B$ can be applied naturally to a function. Using Assumptions 3.1 and 3.2 , we determine that

$$
\begin{aligned}
\mid B( & \left.W^{n+1}-2 W^{n}+W^{n-1}\right) \mid \\
\leq & \left|B\left(e^{n+1}-2 e^{n}+e^{n-1}\right)\right|+\left|B\left(\mathcal{E}^{n+1}-2 \mathcal{E}^{n}+\mathcal{E}^{n-1}\right)\right| \\
& +\left|B\left((u-g)^{n+1}-2(u-g)^{n}+(u-g)^{n-1}\right)\right| \\
\leq & \mathcal{C}\left\{\frac{k^{2}}{h^{4}}\left[\left\|\frac{e^{n+1}-e^{n}}{k}\right\|+\left\|\frac{e^{n}-e^{n-1}}{k}\right\|\right]\right. \\
& \left.+\left\|\frac{\mathcal{E}^{n+1}-\mathcal{E}^{n}}{k}\right\|+\left\|\frac{\mathcal{E}^{n}-\mathcal{E}^{n-1}}{k}\right\|+\left(\int_{t^{n-1}}^{t^{n+1}}\left\|(u-g)_{t t}\right\|_{2}^{2} d t\right)^{1 / 2} k^{3 / 2}\right\} .
\end{aligned}
$$


We remark that the last term above was easily estimated to be $\mathcal{O}\left(k^{2}\right)$, which is all we need for our error estimate. However, it should be $\mathcal{O}\left(k^{3}\right)$, as noted above, but this is not so easy to prove rigorously in the finite element case.

Combining (3.4) and (3.5), we obtain

$$
\begin{aligned}
c_{*} \| & \frac{e^{n+1}-e^{n}}{k} \|^{2} k+\left(a \nabla e^{n+1}, \nabla e^{n+1}\right)-\left(a \nabla e^{n}, \nabla e^{n}\right) \\
\leq & \mathcal{C}\left|B\left(W^{n+1}-2 W^{n}+W^{n-1}\right)\right|^{2} k \\
\leq & \mathcal{C}\left\{\left(\frac{k}{h^{2}}\right)^{4}\left[\left\|\frac{e^{n+1}-e^{n}}{k}\right\|^{2} k+\left\|\frac{e^{n}-e^{n-1}}{k}\right\|^{2} k\right]\right. \\
& \left.+\left\|\frac{\mathcal{E}^{n+1}-\mathcal{E}^{n}}{k}\right\|^{2} k+\left\|\frac{\mathcal{E}^{n}-\mathcal{E}^{n-1}}{k}\right\|^{2} k+\int_{t^{n-1}}^{t^{n+1}}\left\|(u-g)_{t t}\right\|_{2}^{2} d t k^{4}\right\} .
\end{aligned}
$$

Summing on $n$ from 1 to $m-1$, we see that

$$
\begin{aligned}
c_{*} \sum_{n=1}^{m-1}\left\|\frac{e^{n+1}-e^{n}}{k}\right\|^{2} k+\left(a \nabla e^{m}, \nabla e^{m}\right) \\
\leq\left(a \nabla e^{1}, \nabla e^{1}\right)+\mathcal{C}\left\{\left(\frac{k}{h^{2}}\right)^{4} \sum_{n=1}^{m-1}\left\|\frac{e^{n+1}-e^{n}}{k}\right\|^{2} k+\left\|\frac{e^{1}-e^{0}}{k}\right\|^{2} k\right. \\
\left.\quad+\sum_{n=0}^{m-1}\left\|\frac{\mathcal{E}^{n+1}-\mathcal{E}^{n}}{k}\right\|^{2} k+\int_{0}^{T}\left\|(u-g)_{t t}\right\|_{2}^{2} d t k^{4}\right\} .
\end{aligned}
$$

For $k / h^{2}$ sufficiently small, we can remove the second term on the right-hand side, and the estimation of the error generated by the AD-M method is reduced to bounding the errors in $w_{h}^{0}$ and $w_{h}^{1}$. With Assumption 3.3, this gives us two of the convergence results stated below.

Theorem 3.1 Under Assumptions 3.1-3.3, the solution $w_{h}^{n}$ of the AD-M (2.11)-(2.13) converges to the solution $u$ of (1.1)-(1.3) in the sense that the error $\delta^{n}=u^{n}-w_{h}^{n}$ satisfies

$$
\begin{aligned}
& \left(\sum_{n=0}^{N-1}\left\|\frac{\delta^{n+1}-\delta^{n}}{k}\right\|^{2} k\right)^{1 / 2}+\max _{1 \leq m \leq N}\left\|\delta^{m}\right\| \\
& \quad \leq \mathcal{C}\left\{h^{s}+k^{3-2 \theta}+\left\|u_{h}^{1}-w_{h}^{1}\right\|_{1}+\left\|\frac{\delta^{1}-\delta^{0}}{k}\right\| \sqrt{k}\right\}, \\
& \max _{1 \leq m \leq N}\left\|\delta^{m}\right\|_{1} \leq \mathcal{C}\left\{h^{s-1}+k^{3-2 \theta}+\left\|u_{h}^{1}-w_{h}^{1}\right\|_{1}+\left\|\frac{\delta^{1}-\delta^{0}}{k}\right\| \sqrt{k}\right\},
\end{aligned}
$$

provided that $k$ and $k / h^{2}$ are sufficiently small, wherein $\mathcal{C}$ depends on the smoothness of $u$, $a, c$, and $g$, but not on $h$ or $k$, and $u_{h}$ is the solution to the unmodified method (2.2)-(2.3).

It remains to prove the estimate on $\max \left\|\delta^{m}\right\|$. Returning to (3.3), we take test vector $\theta E^{n+1}+(1-\theta) E^{n}$ to obtain, for $n=1,2, \ldots, N-1$,

$$
\begin{aligned}
& \left(c \frac{e^{n+1}-e^{n}}{k}, \theta e^{n+1}+(1-\theta) e^{n}\right)+\left(a \nabla\left[\theta e^{n+1}+(1-\theta) e^{n}\right], \nabla\left[\theta e^{n+1}+(1-\theta) e^{n}\right]\right) \\
& =B\left(W^{n+1}-2 W^{n}+W^{n-1}\right) \cdot\left(\theta E^{n+1}+(1-\theta) E^{n}\right) .
\end{aligned}
$$


Again, for either $\theta=1$ or $\theta=1 / 2$, we can estimate

$$
\begin{aligned}
\frac{1}{2}( & \left.\left\|c^{1 / 2} e^{n+1}\right\|^{2}-\left\|c^{1 / 2} e^{n}\right\|^{2}\right) \\
\leq & \mathcal{C}\left|B\left(W^{n+1}-2 W^{n}+W^{n-1}\right)\right|\left\|\theta e^{n+1}+(1-\theta) e^{n}\right\| k \\
\leq & \mathcal{C}\left\{\left|B\left(W^{n+1}-2 W^{n}+W^{n-1}\right)\right|^{2} k+\left(\left\|e^{n+1}\right\|^{2}+\left\|e^{n}\right\|^{2}\right) k\right\} \\
\leq & \mathcal{C}\left\{\left(\left\|e^{n+1}\right\|^{2}+\left\|e^{n}\right\|^{2}\right) k+\left\|\frac{e^{n+1}-e^{n}}{k}\right\|^{2} k+\left\|\frac{e^{n}-e^{n-1}}{k}\right\|^{2} k\right. \\
& \left.+\left\|\frac{\mathcal{E}^{n+1}-\mathcal{E}^{n}}{k}\right\|^{2} k+\left\|\frac{\mathcal{E}^{n}-\mathcal{E}^{n-1}}{k}\right\|^{2} k+\int_{t^{n-1}}^{t^{n+1}}\left\|(u-g)_{t t}\right\|_{2}^{2} d t k^{4}\right\},
\end{aligned}
$$

using (3.5) again. Now sum on $n$ from 1 to $m-1$ to obtain that

$$
\begin{aligned}
\left\|c^{1 / 2} e^{m}\right\|^{2} \leq & \left\|c^{1 / 2} e^{1}\right\|^{2}+\mathcal{C}\left\{\sum_{n=1}^{m}\left\|e^{n}\right\|^{2} k\right. \\
& \left.+\sum_{n=0}^{m-1}\left\|\frac{e^{n+1}-e^{n}}{k}\right\|^{2} k+\sum_{n=0}^{m-1}\left\|\frac{\mathcal{E}^{n+1}-\mathcal{E}^{n}}{k}\right\|^{2} k+\int_{0}^{T}\left\|(u-g)_{t t}\right\|_{2}^{2} d t k^{4}\right\} .
\end{aligned}
$$

We can remove the second term on the right-hand side using the Gronwall inequality, provided that $k$ is sufficiently small. The third term on the right-hand side is estimated using the first result of the theorem. Assumption 3.3 completes the proof in the finite element case. The finite difference case is similar to the above, only simpler, since it is trivial to apply $B$ to a set of finite difference points of the true solution.

\section{The mixed finite element formulation.}

We rewrite (1.1)-(1.3) in mixed form by defining

$$
\mathbf{q}=-a \nabla u
$$

and then, with $\alpha(x)=1 / a(x)$, we have

$$
\begin{aligned}
& \alpha \mathbf{q}+\nabla u=0, \quad x \in \Omega, \quad 0<t \leq T, \\
& c u_{t}+\nabla \cdot \mathbf{q}=f, \quad x \in \Omega, \quad 0<t \leq T, \\
& u=g, \quad x \in \partial \Omega, \quad 0<t \leq T, \\
& u=u_{0}, \quad x \in \Omega, \quad t=0 .
\end{aligned}
$$

Define the function spaces

$$
\begin{aligned}
& \mathbf{V}=H(\operatorname{div} ; \Omega)=\left\{\mathbf{q} \in\left(L^{2}(\Omega)\right)^{d}: \nabla \cdot \mathbf{q} \in L^{2}(\Omega)\right\}, \\
& W=L^{2}(\Omega) .
\end{aligned}
$$

If (4.2) is tested by a function in $\mathbf{V}$ and (4.3) is tested by a function in $W$, the weak form of (4.2)-(4.4) of interest for the mixed method results; that is, we find for each time $(\mathbf{q}, u) \in$ $V \times W$ such that

$$
\begin{aligned}
(\alpha \mathbf{q}, \mathbf{v})-(\nabla \cdot \mathbf{v}, u) & =-\langle g, \mathbf{v} \cdot \nu\rangle, & \mathbf{v} \in \mathbf{V} \\
\left(c u_{t}, w\right)+(\nabla \cdot \mathbf{q}, w) & =(f, w), & w \in W
\end{aligned}
$$


where the inner-product $(\cdot, \cdot)$ is taken in $W$ or $W^{d}$, as appropriate, $\langle\cdot, \cdot\rangle$ is the inner-product in $L^{2}(\partial \Omega)$, and $\nu$ is the outer unit normal to $\partial \Omega$.

The mixed finite element method approximates the solution in a properly chosen subspace $\mathbf{V}_{h} \times W_{h}$ of $\mathbf{V} \times W$ (see, e.g., [14]). In semi-discrete form (i.e., discretizing space only), we seek $\left(\mathbf{q}_{h}(t), u_{h}(t)\right) \in \mathbf{V}_{h} \times W_{h}$ such that

$$
\begin{aligned}
\left(\alpha \mathbf{q}_{h}, \mathbf{v}\right)-\left(\nabla \cdot \mathbf{v}, u_{h}\right) & =-\langle g, \mathbf{v} \cdot \nu\rangle, & & \mathbf{v} \in \mathbf{V}_{h}, \\
\left(c u_{h, t}, w\right)+\left(\nabla \cdot \mathbf{q}_{h}, w\right) & =(f, w), & & w \in W_{h} .
\end{aligned}
$$

\section{$5 \quad$ AD-M for mixed finite elements.}

We derive our AD-M method only for the case that $d=2$; the extension to $d=3$ is straightforward. We assume that $\mathbf{V}_{h} \times W_{h}$ is the Raviart-Thomas space $R T_{s-1}$ [14] of index $s-1 \geq 0$, though spaces with similar properties could be used instead, such as the BrezziDouglas-Fortin-Marini spaces [2]. The usual basis for $\mathbf{V}_{h}$,

$$
\mathbf{V}_{h}=\operatorname{span}_{i, j}\left\{\mathbf{v}_{x, i}, \mathbf{v}_{y, j}\right\}
$$

has the properties that the vector function $\mathbf{v}_{x, i}$ has a vanishing $y$-component, is supported in at most two grid elements sharing an edge with normal in the $x$-direction, and is discontinuous in the $y$-direction; similarly, $\mathbf{v}_{y, j}$ has a vanishing $x$-component, is supported in at most two grid elements sharing an edge with normal in the $y$-direction, and is discontinuous in the $x$-direction. The usual basis for $W_{h}$,

$$
W_{h}=\operatorname{span}_{\ell}\left\{w_{\ell}\right\}
$$

is piecewise discontinuous over the grid.

We now reduce (4.8)-(4.9) to a system of linear equations. First consider the matrix $A$ arising from the form $\left(\alpha \mathbf{v}_{1}, \mathbf{v}_{2}\right)$, for two basis functions of $\mathbf{V}_{h}$. Any mixture of $x$ and $y$ basis functions results in

$$
\left(\alpha \mathbf{v}_{x, i}, \mathbf{v}_{y, j}\right)=0
$$

so $A$ is block diagonal with blocks

$$
\begin{aligned}
& A_{x, i_{1}, i_{2}}=\left(\alpha \mathbf{v}_{x, i_{1}}, \mathbf{v}_{x, i_{2}}\right) \\
& A_{y, j_{1}, j_{2}}=\left(\alpha \mathbf{v}_{y, j_{1}}, \mathbf{v}_{y, j_{2}}\right) .
\end{aligned}
$$

Note that both $A_{x}$ and $A_{y}$ are invertible. Moreover, with the appropriate ordering, these matrices are banded with bands densely concentrated around the diagonal. For the $x$-basis functions, we use standard $(i, j)$ ordering of the elements, with $i$ advancing fastest. Starting from $(1,1)$, we progress through the grid, numbering all $\mathbf{v}_{x, \ell}$ with support in $(i, j)$. However, for the $y$-basis functions, we use $(i, j)$ ordering of the elements with $j$ advancing fastest. Thus these matrices have just a few bands near the diagonal, and so it is easy to solve linear subsystems involving $A_{x}$ and $A_{y}$.

With any ordering of the elements, we also have the nonsingular, block diagonal matrix $C$ defined by

$$
C_{\ell_{1}, \ell_{2}}=\left(c w_{\ell_{1}}, w_{\ell_{2}}\right)
$$


Finally, let

$$
\begin{aligned}
& B_{x, i, \ell}=\left(w_{\ell}, \nabla \cdot \mathbf{v}_{x, i}\right), \\
& B_{y, j, \ell}=\left(w_{\ell}, \nabla \cdot \mathbf{v}_{y, j}\right),
\end{aligned}
$$

which are sparse, but not particularly well structured.

Let us expand the solution in the basis as

$$
\begin{aligned}
& u_{h}(x, y, t)=\sum_{\ell} z_{\ell}(t) w_{\ell}(x, y), \\
& \mathbf{q}_{h}(x, y, t)=\sum_{i} \mu_{i}(t) \mathbf{v}_{x, i}(x, y)+\sum_{j} \lambda_{j}(t) \mathbf{v}_{y, j}(x, y) .
\end{aligned}
$$

Then the equations (4.8)-(4.9) reduce to the system of linear equations

$$
\begin{aligned}
A_{x} \mu-B_{x} z & =G_{x}, \\
A_{y} \lambda-B_{y} z & =G_{y}, \\
C z_{t}+B_{x}^{T} \mu+B_{y}^{T} \lambda & =F,
\end{aligned}
$$

where

$$
\begin{aligned}
& G_{x, i}=-\left\langle g, \mathbf{v}_{x, i} \cdot \nu\right\rangle, \\
& G_{y, j}=-\left\langle g, \mathbf{v}_{y, j} \cdot \nu\right\rangle, \\
& F_{\ell}=\left(f, w_{\ell}\right) .
\end{aligned}
$$

\subsection{Backward Euler and Crank-Nicolson time discretization.}

When we employ backward Euler $(\theta=1)$ or Crank-Nicolson $(\theta=1 / 2)$ time discretization, we begin with some approximation of $z^{0}$ and define $\mu^{0}$ and $\lambda^{0}$ from

$$
\begin{aligned}
& A_{x} \mu^{0}-B_{x} z^{0}=G_{x}^{0}, \\
& A_{y} \lambda^{0}-B_{y} z^{0}=G_{y}^{0},
\end{aligned}
$$

and then, for $n=0,1, \ldots, N-1$, our system becomes

$$
\begin{aligned}
A_{x} \mu^{n+1}-B_{x} z^{n+1} & =G_{x}^{n+1}, \\
A_{y} \lambda^{n+1}-B_{y} z^{n+1} & =G_{y}^{n+1}, \\
C \frac{z^{n+1}-z^{n}}{k}+B_{x}^{T}\left(\theta \mu^{n+1}+(1-\theta) \mu^{n}\right)+B_{y}^{T}\left(\theta \lambda^{n+1}+(1-\theta) \lambda^{n}\right) & =F^{n+\theta} .
\end{aligned}
$$

This is an indefinite saddle-point problem, and, therefore, generally difficult to solve.

Now let

$$
M_{x}=B_{x}^{T} A_{x}^{-1} B_{x} \quad \text { and } \quad M_{y}=B_{y}^{T} A_{y}^{-1} B_{y} .
$$

By solving (5.6) and (5.7) or (5.8) and (5.9) for $\mu^{n}$ and $\lambda^{n}$, we reduce (5.10) to

$$
C \frac{z^{n+1}-z^{n}}{k}+\left(M_{x}+M_{y}\right)\left(\theta z^{n+1}+(1-\theta) z^{n}\right)=\mathcal{F}^{n+1}
$$

where

$$
\mathcal{F}^{n+1}=F^{n+\theta}-B_{x}^{T} A_{x}^{-1}\left(\theta G_{x}^{n+1}+(1-\theta) G_{x}^{n}\right)-B_{y}^{T} A_{y}^{-1}\left(\theta G_{y}^{n+1}+(1-\theta) G_{y}^{n}\right) .
$$

Now (5.11) is positive definite, but unfortunately, $M_{x}+M_{y}$ is a full matrix, and so it is still difficult to solve. 


\subsection{An Uzawa mixed AD method.}

An efficiently computable Uzawa AD algorithm (see [9]) iterates on $n=0,1, \ldots, N-1$ as follows:

$x$-sweep:

$$
\begin{aligned}
A_{x} \mu^{n, 1}-B_{x} z^{n, 1} & =G_{x}^{n+1}, \\
C \frac{z^{n, 1}-z^{n}}{k}+B_{x}^{T}\left(\theta \mu^{n, 1}+(1-\theta) \mu^{n}\right)+B_{y}^{T} \lambda^{n} & =F^{n+\theta}
\end{aligned}
$$

$y$-sweep:

$$
\begin{aligned}
A_{y} \lambda^{n+1}-B_{y} z^{n+1} & =G_{y}^{n+1}, \\
C \frac{z^{n+1}-z^{n}}{k}+B_{x}^{T}\left(\theta \mu^{n, 1}+(1-\theta) \mu^{n}\right)+B_{y}^{T}\left(\theta \lambda^{n+1}+(1-\theta) B_{y}^{T} \lambda^{n}\right) & =F^{n+\theta}
\end{aligned}
$$

corrector step:

$$
A_{x} \mu^{n+1}-B_{x} z^{n+1}=G_{x}^{n+1} .
$$

Eliminating $\mu^{n}$ and $\lambda^{n}$, the Uzawa AD algorithm becomes

$$
\begin{array}{r}
C \frac{z^{n, 1}-z^{n}}{k}+M_{x}\left(\theta z^{n, 1}+(1-\theta) z^{n}\right)+M_{y} z^{n}=\mathcal{F}^{n, 1} \\
C \frac{z^{n+1}-z^{n}}{k}+M_{x}\left(\theta z^{n, 1}+(1-\theta) z^{n}\right)+M_{y}\left(\theta z^{n+1}+(1-\theta) z^{n}\right)=\mathcal{F}^{n+1},
\end{array}
$$

where

$$
\mathcal{F}^{n, 1}=F^{n+\theta}-B_{x}^{T} A_{x}^{-1}\left(\theta G_{x}^{n+1}+(1-\theta) G_{x}^{n}\right)-B_{y}^{T} A_{y}^{-1} G_{y}^{n} .
$$

Subtract (5.18) from (5.19), multiply the result by $\theta k M_{x} C^{-1}$, and combine with (5.19) to obtain the single equation

$$
\begin{aligned}
& C \frac{z^{n+1}-z^{n}}{k}+\left(M_{x}+M_{y}\right)\left(\theta z^{n+1}+(1-\theta) z^{n}\right)+\theta^{2} k M_{x} C^{-1} M_{y}\left(z^{n+1}-z^{n}\right) \\
& \quad=\mathcal{F}^{n+1}-\theta^{2} k M_{x} C^{-1} B_{y}^{T} A_{y}^{-1}\left(G_{y}^{n+1}-G_{y}^{n}\right) .
\end{aligned}
$$

Comparing this with (5.11) shows that the splitting error is

$$
\theta^{2} k M_{x} C^{-1}\left[M_{y}\left(z^{n+1}-z^{n}\right)+B_{y}^{T} A_{y}^{-1}\left(G_{y}^{n+1}-G_{y}^{n}\right)\right]=\theta^{2} k M_{x} C^{-1} B_{y}^{T}\left(\lambda^{n+1}-\lambda^{n}\right),
$$

which is $\mathcal{O}\left(k^{2}\right)$ for a sufficiently smooth solution $u$ and boundary condition $g$.

\subsection{A new Uzawa mixed AD-M method.}

The equations (5.21) are not efficiently computable, but they do illuminate the splitting error and suggest, similar to [7, (2.9)], that we can reduce it to $\mathcal{O}\left(k^{3}\right)$ by adding terms to make the splitting error equal to

$$
\begin{aligned}
& \theta^{2} k M_{x} C^{-1}\left[M_{y}\left(z^{n+1}-2 z^{n}+z^{n-1}\right)+B_{y}^{T} A_{y}^{-1}\left(G_{y}^{n+1}-2 G_{y}^{n}+G_{y}^{n-1}\right)\right] \\
& =\theta^{2} k M_{x} C^{-1} B_{y}^{T}\left(\lambda^{n+1}-2 \lambda^{n}+\lambda^{n-1}\right)=\mathcal{O}\left(k^{3}\right)
\end{aligned}
$$


Then the local splitting error would be higher order in $k$ than the local error for the backward Euler or Crank-Nicolson approximation, and also for the local splitting error of the AD method itself.

Although $A_{x}$ and $A_{y}$ have bands concentrated near the diagonal, their inverses may be full, so to avoid computing the inverse of $A_{x}$ and $A_{y}$, we propose the efficiently computable algorithm, for $n=1,2, \ldots, N-1$,

$x$-sweep:

$$
\begin{aligned}
A_{x} \mu^{n, 1}-B_{x} z^{n, 1}+\theta k B_{x} C^{-1} B_{y}^{T}\left(\lambda^{n}-\lambda^{n-1}\right) & =G_{x}^{n+1}, \\
C \frac{z^{n, 1}-z^{n}}{k}+B_{x}^{T}\left(\theta \mu^{n, 1}+(1-\theta) \mu^{n}\right)+B_{y}^{T} \lambda^{n} & =F^{n+\theta},
\end{aligned}
$$

$y$-sweep:

$$
\begin{aligned}
A_{y} \lambda^{n+1}-B_{y} z^{n+1} & =G_{y}^{n+1}, \\
C \frac{z^{n+1}-z^{n}}{k}+B_{x}^{T}\left(\theta \mu^{n, 1}+(1-\theta) \mu^{n}\right)+B_{y}^{T}\left(\theta \lambda^{n+1}+(1-\theta) \lambda^{n}\right) & =F^{n+\theta},
\end{aligned}
$$

corrector step:

$$
A_{x} \mu^{n+1}-B_{x} z^{n+1}=G_{x}^{n+1} .
$$

After some manipulation, we have

$$
\begin{aligned}
& C \frac{z^{n+1}-z^{n}}{k}+\left(M_{x}+M_{y}\right)\left(\theta z^{n+1}+(1-\theta) z^{n}\right)+\theta^{2} k M_{x} C^{-1} M_{y}\left(z^{n+1}-2 z^{n}+z^{n-1}\right) \\
& \quad=\mathcal{F}^{n+1}-\theta^{2} k M_{x} C^{-1} B_{y}^{T} A_{y}^{-1}\left(G_{y}^{n+1}-2 G_{y}^{n}+G_{y}^{n-1}\right)
\end{aligned}
$$

which has the promised $\mathcal{O}\left(k^{3}\right)$ splitting error.

\section{Convergence of mixed AD-M.}

We suppose that $\mathbf{V}_{h} \times W_{h}$ approximates $\mathbf{V} \times W$ as in the case of $R T_{s-1}$, that is,

$$
\min _{\mathbf{v} \in \mathbf{V}_{h}}\|\mathbf{q}-\mathbf{v}\| \leq \mathcal{C}\|\mathbf{q}\|_{s} h^{s} \text { and } \min _{w \in W_{h}}\|u-w\| \leq \mathcal{C}\|u\|_{s} h^{s} .
$$

All the usual mixed spaces satisfy the property that $\nabla \cdot \mathbf{V}_{h}=W_{h}$, and they each have a linear projection operator $\pi: \mathbf{V} \cap\left(L^{p}(\Omega)\right)^{d} \rightarrow \mathbf{V}_{h}$, where $p>2$, such that

$$
\begin{aligned}
& \|\mathbf{v}-\pi \mathbf{v}\| \leq \mathcal{C}\|\mathbf{v}\|_{s} h^{s}, \\
& (\nabla \cdot(\mathbf{v}-\pi \mathbf{v}), w)=0, \quad w \in W_{h}, \\
& \|\nabla \cdot(\mathbf{v}-\pi \mathbf{v})\| \leq \mathcal{C}\|\nabla \cdot \mathbf{v}\|_{s} h^{s} .
\end{aligned}
$$

We also let $\mathcal{P}$ be the linear orthogonal projection operator of $L^{2}(\Omega)$ onto $W_{h}$. We will also need below $\mathcal{P}_{0}$, which is the linear orthogonal projection operator of $L^{2}(\Omega)$ onto the space of discontinuous constants (i.e., the scalar space of $R T_{0}$ ). Trivially,

$$
\left\|c-\mathcal{P}_{0} c\right\|_{L^{\infty}} \leq \mathcal{C}\|\nabla c\|_{L^{\infty}} h
$$

where $\|\cdot\|_{L^{\infty}}$ is the $L^{\infty}$-norm. 
Mixed methods on rectangles have many interesting and important superconvergence properties. For example, it is known $[12,10,11]$ that the weighted $L^{2}$-projection $\mathcal{P}_{\mathbf{V}}^{\alpha}$, defined by

$$
\left(\alpha\left(\mathbf{v}-\mathcal{P}_{\mathbf{V}}^{\alpha} \mathbf{v}\right), \tilde{\mathbf{v}}\right)=0, \quad \tilde{\mathbf{v}} \in \mathbf{V}_{h}
$$

has the property that

$$
\left\|\pi \mathbf{v}-\mathcal{P}_{\mathbf{V}}^{\alpha} \mathbf{v}\right\| \leq \mathcal{C}\|\mathbf{v}\|_{s+1} h^{s+1} .
$$

Recalling (5.1)-(5.2), we have from (4.6) and either (5.6)-(5.7) or (5.26), (5.24) that the errors $e^{n}=u^{n}-u_{h}^{n}$ and $\sigma^{n}=\mathbf{q}^{n}-\mathbf{q}_{h}^{n}$ satisfy, for each $n=0,1, \ldots, N$,

$$
\left(\alpha \pi \sigma^{n}, \mathbf{v}\right)-\left(\nabla \cdot \mathbf{v}, \mathcal{P} e^{n}\right)=\left(\alpha\left(\pi \mathbf{q}^{n}-\mathbf{q}^{n}, \mathbf{v}\right), \quad \mathbf{v} \in \mathbf{V}_{h}\right.
$$

Solving either (5.6)-(5.7) or (5.26), (5.24) for $\mu^{n}$ and $\lambda^{n}$, we can rewrite (5.27) as

$$
\begin{gathered}
C \frac{z^{n+1}-z^{n}}{k}+B_{x}^{T}\left(\theta \mu^{n+1}+(1-\theta) \mu^{n}\right)+B_{y}^{T}\left(\theta \lambda^{n+1}+(1-\theta) \lambda^{n}\right) \\
=\theta F^{n+1}+(1-\theta) F^{n}-\theta^{2} k M_{x} C^{-1} B_{y}^{T}\left(\lambda^{n+1}-2 \lambda^{n}+\lambda^{n-1}\right) .
\end{gathered}
$$

From (4.7) at times $t^{n}$ and $t^{n+1}$, then, we obtain for $w_{i} \in W_{h}$ that

$$
\begin{aligned}
&(c \mathcal{P}\left.\frac{e^{n+1}-e^{n}}{k}, w_{i}\right)+\left(\nabla \cdot\left[\theta \pi \sigma^{n+1}+(1-\theta) \pi \sigma^{n}\right], w_{i}\right) \\
&= \theta^{2} k\left(M_{x} C^{-1} B_{y}^{T}\left(\lambda^{n+1}-2 \lambda^{n}+\lambda^{n-1}\right)\right)_{i} \\
& \quad+\left(c\left[\mathcal{P} \frac{u^{n+1}-u^{n}}{k}-\theta u_{t}^{n+1}-(1-\theta) u_{t}^{n}\right], w_{i}\right),
\end{aligned}
$$

wherein we introduced $\pi$ trivially using (6.3).

We bound the splitting error as follows. First note that

$$
\begin{aligned}
B_{y}^{T} \lambda & =M_{y} z+B_{y}^{T} A_{y}^{-1} G_{y} \\
& =-M_{y} E+M_{y} U+B_{y}^{T} A_{y}^{-1} G_{y} \\
& =-M_{y} E+B_{y}^{T} A_{y}^{-1}\left(B_{y} U+G_{y}\right),
\end{aligned}
$$

where $E$ and $U$ are the vectors of finite element coefficients of $\mathcal{P} e$ and $\mathcal{P} u$, respectively. Now, if we take test function $\mathbf{v}_{y, j}$ in (4.6) and let $\mathcal{Q}$ be the $\alpha$-weighted linear orthogonal projection of $\left(L^{2}(\Omega)\right)^{2}$ onto $\mathbf{V}_{h}$, we see that

$$
\left(\alpha \mathcal{Q q}, \mathbf{v}_{y, j}\right)-\left(\nabla \cdot \mathbf{v}_{y, j}, \mathcal{P} u\right)=-\left\langle g, \mathbf{v}_{y, j} \cdot \nu\right\rangle
$$

and, with $Q$ being the vector of finite element coefficients of $\mathcal{Q} \mathbf{q}$,

$$
A_{y} Q-B_{y} U=G_{y}
$$

Combining, we have that

$$
B_{y}^{T} \lambda=-M_{y} E+B_{y}^{T} Q
$$


The second step in bounding the splitting error is to note that $\left\|A_{x}\right\|+\left\|A_{y}\right\|+\|C\| \leq \mathcal{C}$ and $\left\|A_{x}^{-1}\right\|+\left\|A_{y}^{-1}\right\|+\left\|C^{-1}\right\| \leq \mathcal{C}$, and that we have only $\left\|B_{x}\right\|+\left\|B_{y}\right\| \leq \mathcal{C} h^{-1}$. Thus,

$$
\begin{aligned}
& \left|\theta^{2} k M_{x} C^{-1} B_{y}^{T}\left(\lambda^{n+1}-2 \lambda^{n}+\lambda^{n-1}\right)\right| \\
& \quad \leq\left|k M_{x} C^{-1} M_{y}\left(E^{n+1}-2 E^{n}+E^{n-1}\right)\right|+\left|k M_{x} C^{-1} B_{y}^{T}\left(Q^{n+1}-2 Q^{n}+Q^{n-1}\right)\right| \\
& \quad \leq \mathcal{C} k h^{-2}\left\{h^{-2}\left\|\mathcal{P}\left(e^{n+1}-2 e^{n}+e^{n-1}\right)\right\|+\left\|\partial_{2}\left(\mathcal{Q} q_{2}^{n+1}-2 \mathcal{Q} q_{2}^{n}+\mathcal{Q} q_{2}^{n-1}\right)\right\|\right\} \\
& \quad \leq \mathcal{C} \frac{k}{h^{2}}\left\{\frac{k}{h^{2}}\left(\left\|\mathcal{P} \frac{e^{n+1}-e^{n}}{k}\right\|+\left\|\mathcal{P} \frac{e^{n}-e^{n-1}}{k}\right\|\right)+k \int_{t^{n-1}}^{t^{n+1}}\left\|\partial_{2}\left(\mathcal{Q} q_{2}\right)_{t t}\right\| d t\right\} \\
& \quad \leq \mathcal{C} \frac{k}{h^{2}}\left\{\frac{k}{h^{2}}\left(\left\|\mathcal{P} \frac{e^{n+1}-e^{n}}{k}\right\|+\left\|\mathcal{P} \frac{e^{n}-e^{n-1}}{k}\right\|\right)+k^{3 / 2}\left(\int_{t^{n-1}}^{t^{n+1}}\left\|q_{2, t t}\right\|_{1}^{2} d t\right)^{1 / 2}\right\},
\end{aligned}
$$

using the stability of the $L^{2}$-projection $\mathcal{Q}$ in $H^{1}$.

The overall analysis of (6.8) proceeds much as in the nonmixed case. First take the difference of (6.6) at times $t^{n+1}$ and $t^{n}$, and then choose the test function $\mathbf{v}=\theta \pi \sigma^{n+1}+(1-$ $\theta) \pi \sigma^{n}$. Combine the result with $w=\mathcal{P}\left(e^{n+1}-e^{n}\right)$ in (6.8), and obtain that

$$
\begin{aligned}
(c \mathcal{P} & \left.\frac{e^{n+1}-e^{n}}{k}, \mathcal{P}\left(e^{n+1}-e^{n}\right)\right)+\left(\alpha\left(\pi \sigma^{n+1}-\pi \sigma^{n}\right), \theta \pi \sigma^{n+1}+(1-\theta) \pi \sigma^{n}\right) \\
= & \theta^{2} k M_{x} C^{-1} B_{y}^{T}\left(\lambda^{n+1}-2 \lambda^{n}+\lambda^{n-1}\right) \cdot\left(E^{n+1}-E^{n}\right) \\
& +\left(\mathcal{P}\left\{c\left[\mathcal{P} \frac{u^{n+1}-u^{n}}{k}-\theta u_{t}^{n+1}-(1-\theta) u_{t}^{n}\right]\right\}, \mathcal{P}\left(e^{n+1}-e^{n}\right)\right) \\
& +\left(\alpha\left(\pi \mathbf{q}^{n+1}-\mathcal{P}_{\mathbf{V}}^{\alpha} \mathbf{q}^{n+1}-\pi \mathbf{q}^{n}+\mathcal{P}_{\mathbf{V}}^{\alpha} \mathbf{q}^{n}\right), \theta \pi \sigma^{n+1}+(1-\theta) \pi \sigma^{n}\right),
\end{aligned}
$$

wherein we introduced the operators $\mathcal{P}$ and $\mathcal{P}_{\mathbf{V}}^{\alpha}$. Again assuming that $k / h^{2}$ is sufficiently small, after some manipulation similar to that in $\S 3$, we obtain

$$
\begin{aligned}
\frac{1}{2} \| & \sqrt{c} \mathcal{P} \frac{e^{n+1}-e^{n}}{k} \|^{2} k+\frac{1}{2}\left(\left\|\sqrt{\alpha} \pi \sigma^{n+1}\right\|^{2}-\left\|\sqrt{\alpha} \pi \sigma^{n}\right\|^{2}\right) \\
\leq & \mathcal{C}\left\{\left(\frac{k}{h^{2}}\right)^{4}\left[\left\|\mathcal{P} \frac{e^{n+1}-e^{n}}{k}\right\|^{2} k+\left\|\mathcal{P} \frac{e^{n}-e^{n-1}}{k}\right\|^{2} k\right]\right. \\
& +k^{4} \int_{t^{n-1}}^{t^{n+1}}\left\|q_{2, t t}\right\|_{1}^{2} d t+\left\|\mathcal{P}\left(c\left[\mathcal{P} \frac{u^{n+1}-u^{n}}{k}-\theta u_{t}^{n+1}-(1-\theta) u_{t}^{n}\right]\right)\right\|^{2} k \\
& \left.+\left\|\left(\mathcal{P}_{\mathbf{V}}^{\alpha} \mathbf{q}^{n+1}-\pi \mathbf{q}^{n+1}\right)-\left(\mathcal{P}_{\mathbf{V}}^{\alpha} \mathbf{q}^{n}-\pi \mathbf{q}^{n}\right)\right\|^{2} k^{-1}+\left\|\sigma^{n+1}\right\|^{2} k+\left\|\sigma^{n}\right\|^{2} k\right\} .
\end{aligned}
$$

For the time truncation error, we expand

$$
c=\left(c-\mathcal{P}_{0} c\right)+\mathcal{P}_{0} c
$$


and bound

$$
\begin{aligned}
\| \mathcal{P} & \left(\left(c-\mathcal{P}_{0} c\right)\left[\mathcal{P} \frac{u^{n+1}-u^{n}}{k}-\theta u_{t}^{n+1}-(1-\theta) u_{t}^{n}\right]\right) \|^{2} k \\
\leq & \left\|c-\mathcal{P}_{0} c\right\|_{L^{\infty}}\left\{\left\|\mathcal{P}\left(\frac{u^{n+1}-u^{n}}{k}-\theta u_{t}^{n+1}-(1-\theta) u_{t}^{n}\right)\right\|\right. \\
& \left.+\theta\left\|u_{t}^{n+1}-\mathcal{P} u_{t}^{n+1}\right\|+(1-\theta)\left\|u_{t}^{n}-\mathcal{P} u_{t}^{n}\right\|\right\}^{2} k \\
\leq & \mathcal{C}\left\{k^{6-4 \theta} h^{2} \int_{t^{n}}^{t^{n+1}}\left\|\partial_{t}^{4-2 \theta} u\right\|^{2} d t+\left(\left\|u_{t}^{n+1}\right\|_{s}^{2}+\left\|u_{t}^{n}\right\|_{s}^{2}\right) h^{2(s+1)} k\right\},
\end{aligned}
$$

since $\mathcal{P}$ is bounded in the $L^{2}$-norm. The remaining term is then

$$
\left\|\mathcal{P}_{0} c \mathcal{P}\left[\frac{u^{n+1}-u^{n}}{k}-\theta u_{t}^{n+1}-(1-\theta) u_{t}^{n}\right]\right\|^{2} k \leq \mathcal{C} k^{6-4 \theta} \int_{t^{n}}^{t^{n+1}}\left\|\partial_{t}^{4-2 \theta} u\right\|^{2} d t .
$$

Moreover, we have that

$$
\begin{aligned}
& \left\|\left(\mathcal{P}_{\mathbf{V}}^{\alpha} \mathbf{q}^{n+1}-\pi \mathbf{q}^{n+1}\right)-\left(\mathcal{P}_{\mathbf{V}}^{\alpha} \mathbf{q}^{n}-\pi \mathbf{q}^{n}\right)\right\|^{2} k^{-1}=\left\|\int_{t^{n}}^{t^{n+1}}\left(\mathcal{P}_{\mathbf{V}}^{\alpha} \mathbf{q}-\pi \mathbf{q}\right)_{t} d t\right\|^{2} k^{-1} \\
& \quad \leq\left(\int_{t^{n}}^{t^{n+1}}\left\|\mathcal{P}_{\mathbf{V}}^{\alpha} \mathbf{q}_{t}-\pi \mathbf{q}_{t}\right\| d t\right)^{2} k^{-1} \leq \int_{t^{n}}^{t^{n+1}}\left\|\mathcal{P}_{\mathbf{V}}^{\alpha} \mathbf{q}_{t}-\pi \mathbf{q}_{t}\right\|^{2} d t \\
& \quad \leq h^{2(s+1)} \int_{t^{n}}^{t^{n+1}}\left\|\mathbf{q}_{t}\right\|_{s+1}^{2} d t
\end{aligned}
$$

Finally, Gronwall's lemma implies that, for $k$ and $k / h^{2}$ sufficiently small,

$$
\begin{aligned}
& \sum_{n=1}^{N-1}\left\|\mathcal{P} \frac{e^{n+1}-e^{n}}{k}\right\|^{2} k+\max _{1 \leq n \leq N}\left\|\pi \sigma^{n}\right\|^{2} \\
& \leq \mathcal{C}\left\{\left\|\pi \sigma^{1}\right\|^{2}+\left\|\mathcal{P} \frac{e^{1}-e^{0}}{k}\right\|^{2} k+k^{4} \int_{0}^{T}\left\|q_{2, t t}\right\|_{1}^{2} d t+k^{6-4 \theta} \int_{0}^{T}\left\|\partial_{t}^{4-2 \theta} u\right\|^{2} d t\right. \\
& \left.\quad+h^{2(s+1)}\left(\sum_{n=1}^{N}\left\|u_{t}^{n}\right\|_{s}^{2} k+\int_{0}^{T}\left\|\mathbf{q}_{t}\right\|_{s+1}^{2} d t\right)\right\} .
\end{aligned}
$$

For our second estimate of (6.8), take the $\theta$-weighted average of (6.6) at times $t^{n+1}$ and $t^{n}$, and choose the test function $\mathbf{v}=\theta \pi \sigma^{n}+(1-\theta) \pi \sigma^{n}$. Combined with (6.8) using $w=\theta \mathcal{P} e^{n+1}+(1-\theta) \mathcal{P} e^{n}$, it follows that

$$
\begin{aligned}
(c \mathcal{P} & \left.\frac{e^{n+1}-e^{n}}{k}, \theta \mathcal{P} e^{n+1}+(1-\theta) \mathcal{P} e^{n}\right) \\
& +\left(\alpha\left(\theta \pi \sigma^{n+1}+(1-\theta) \pi \sigma^{n}\right), \theta \pi \sigma^{n+1}+(1-\theta) \pi \sigma^{n}\right) \\
= & \theta^{2} k M_{x} C^{-1} B_{y}^{T}\left(\lambda^{n+1}-2 \lambda^{n}+\lambda^{n-1}\right) \cdot\left[\theta \mathcal{P} E^{n+1}+(1-\theta) \mathcal{P} E^{n}\right] \\
& +\left(\mathcal{P}\left\{c\left[\mathcal{P} \frac{u^{n+1}-u^{n}}{k}-\theta u_{t}^{n+1}-(1-\theta) u_{t}^{n}\right]\right\}, \theta \mathcal{P} e^{n+1}+(1-\theta) \mathcal{P} e^{n}\right) \\
& +\left(\alpha\left[\theta\left(\pi \mathbf{q}^{n+1}-\mathcal{P}_{\mathbf{V}}^{\alpha} \mathbf{q}^{n+1}\right)+(1-\theta)\left(\pi \mathbf{q}^{n}-\mathcal{P}_{\mathbf{V}}^{\alpha} \mathbf{q}^{n}\right)\right], \theta \pi \sigma^{n+1}+(1-\theta) \pi \sigma^{n}\right),
\end{aligned}
$$


Again after some manipulation, we have that

$$
\begin{aligned}
\frac{1}{2}\left(\left\|\sqrt{c} \mathcal{P} e^{n+1}\right\|^{2}-\left\|\sqrt{c} \mathcal{P} e^{n}\right\|^{2}\right)+\frac{1}{2}\left\|\sqrt{\alpha}\left(\theta \pi \sigma^{n+1}+(1-\theta) \pi \sigma^{n}\right)\right\|^{2} k \\
\leq \mathcal{C}\left\{\left\|\mathcal{P} \frac{e^{n+1}-e^{n}}{k}\right\|^{2} k+\left\|\mathcal{P} \frac{e^{n}-e^{n-1}}{k}\right\|^{2} k+k^{4} \int_{t^{n-1}}^{t^{n+1}}\left\|q_{2, t t}\right\|_{1}^{2} d t\right. \\
\quad+\left\|\mathcal{P}\left(c\left[\mathcal{P} \frac{u^{n+1}-u^{n}}{k}-\theta u_{t}^{n+1}-(1-\theta) u_{t}^{n}\right]\right)\right\|^{2} k+\left\|\mathcal{P} e^{n+1}\right\|^{2} k+\left\|\mathcal{P} e^{n}\right\|^{2} k \\
\left.\quad+\left\|\mathcal{P}_{\mathbf{V}}^{\alpha} \mathbf{q}^{n+1}-\pi \mathbf{q}^{n+1}\right\|^{2} k+\left\|\mathcal{P}_{\mathbf{V}}^{\alpha} \mathbf{q}^{n}-\pi \mathbf{q}^{n}\right\|^{2} k\right\} .
\end{aligned}
$$

Gronwall's lemma and the previous estimate (6.10) implies that

$$
\begin{aligned}
\left.\max _{1 \leq n \leq N}\left\|\mathcal{P} e^{n}\right\|^{2}+\sum_{n=1}^{N-1} \| \theta \pi \sigma^{n+1}+(1-\theta) \pi \sigma^{n}\right) \|^{2} k \\
\leq \mathcal{C}\left\{\left\|\mathcal{P} e^{1}\right\|^{2}+\left\|\pi \sigma^{1}\right\|^{2}+\left\|\mathcal{P} \frac{e^{1}-e^{0}}{k}\right\|^{2} k\right. \\
\quad+k^{4} \int_{0}^{T}\left\|q_{2, t t}\right\|_{1}^{2} d t+k^{6-4 \theta} \int_{0}^{T}\left\|\partial_{t}^{4-2 \theta} u\right\|^{2} d t \\
\left.\quad+h^{2(s+1)}\left(\sum_{n=1}^{N}\left(\left\|u_{t}^{n}\right\|_{s}^{2}+\left\|\mathbf{q}^{n}\right\|_{s+1}^{2}\right) k+\int_{0}^{T}\left\|\mathbf{q}_{t}\right\|_{s+1}^{2} d t\right)\right\} .
\end{aligned}
$$

Our results (6.10) and (6.11) lead to the following theorem.

Theorem 6.1 Assuming (6.1), the solution $\left(u_{h}^{n}, \mathbf{q}_{h}\right)$ of the mixed AD-M (5.22)-(5.26) converges to the solution $(u, \mathbf{q})$ of (4.6)-(4.7) in the sense that the errors $e^{n}=u^{n}-u_{h}^{n}$ and $\sigma^{n}=\mathbf{q}^{n}-\mathbf{q}_{h}^{n}$ satisfy

$$
\begin{aligned}
& \left(\sum_{n=1}^{N-1}\left\|\mathcal{P} \frac{e^{n+1}-e^{n}}{k}\right\|^{2} k\right)^{1 / 2}+\max _{1 \leq n \leq N}\left\|\mathcal{P} e^{n}\right\|+\max _{1 \leq n \leq N}\left\|\pi \sigma^{n}\right\| \\
& \leq \mathcal{C}\left\{\left\|\mathcal{P} e^{1}\right\|+\left\|\pi \sigma^{1}\right\|+\left\|\mathcal{P} \frac{e^{1}-e^{0}}{k}\right\| \sqrt{k}\right. \\
& \quad+k^{2}\left(\int_{0}^{T}\left\|q_{2, t t}\right\|_{1}^{2} d t\right)^{1 / 2}+k^{3-2 \theta}\left(\int_{0}^{T}\left\|\partial_{t}^{4-2 \theta} u\right\|^{2} d t\right)^{1 / 2} \\
& \left.\quad+h^{s+1}\left[\left(\sum_{n=1}^{N}\left(\left\|u_{t}^{n}\right\|_{s}^{2}+\left\|\mathbf{q}^{n}\right\|_{s+1}^{2}\right) k\right)^{1 / 2}+\left(\int_{0}^{T}\left\|\mathbf{q}_{t}\right\|_{s+1}^{2} d t\right)^{1 / 2}\right]\right\}
\end{aligned}
$$

provided that $k$ and $k / h^{2}$ are sufficiently small, wherein $\mathcal{C}$ depends on the smoothness of a, 
$c$, and $g$, but not on $h$ or $k$. Moreover,

$$
\begin{aligned}
\left(\sum_{n=1}^{N-1}\left\|\frac{e^{n+1}-e^{n}}{k}\right\|^{2} k\right)^{1 / 2}+\max _{1 \leq n \leq N}\left\|e^{n}\right\|+\max _{1 \leq n \leq N}\left\|\sigma^{n}\right\| \\
\leq \mathcal{C}\left\{\left\|\mathcal{P} e^{1}\right\|+\left\|\pi \sigma^{1}\right\|+\left\|\mathcal{P} \frac{e^{1}-e^{0}}{k}\right\| \sqrt{k}\right. \\
+k^{2}\left(\int_{0}^{T}\left\|q_{2, t t}\right\|_{1}^{2} d t\right)^{1 / 2}+k^{3-2 \theta}\left(\int_{0}^{T}\left\|\partial_{t}^{4-2 \theta} u\right\|^{2} d t\right)^{1 / 2} \\
+h^{s}\left[\max _{1 \leq n \leq N}\left\|u^{n}\right\|_{s}+\max _{1 \leq n \leq N}\left\|\mathbf{q}^{n}\right\|_{s}\right. \\
\left.\left.+\left(\sum_{n=1}^{N}\left\|u_{t}^{n}\right\|_{s}^{2} k\right)^{1 / 2}+\left(\int_{0}^{T}\left(\left\|u_{t}\right\|_{s}^{2}+\left\|\mathbf{q}_{t}\right\|_{s}^{2}\right) d t\right)^{1 / 2}\right]\right\} .
\end{aligned}
$$

The last estimate follows from the above argument using only $H^{s}$-smoothness (i.e., not invoking superconvergence) and adding the projection errors to the right-hand side.

\section{Numerical results.}

In this section, we present some numerical experiments illustrating the utility of our mixed method for $R T_{0}$ and confirming our theoretical results. We test only the Crank-Nicolson procedures. The errors reported are measured in discrete $L^{2}$-norms. For the scalar solution $u$, this is

$$
\|\mid\|_{L^{\infty}\left(L^{2}\right)}=\max _{n}\left\{\sum_{\ell}\left(e_{\ell}^{n}\right)^{2} h^{2}\right\}^{1 / 2},
$$

where $e_{\ell}^{n}=u^{n}-u_{h}^{n}$ is the error at the center of grid cell $\ell$, wherein $u$ is the exact solution of (4.2)-(4.5) and $u_{h}$ is the approximation from either the full Crank-Nicholson (C-N) system (5.8)-(5.10), the AD method (5.13)-(5.17), or our AD-M method (5.22)-(5.26). This norm is $\mathcal{O}\left(h^{2}\right)$ close to $\|\mathcal{P} e\|$, and so it should exhibit superconvergence of order $\mathcal{O}\left(h^{2}+k^{2}\right)$.

We also report the errors

$$
\left\|e_{t}\right\|_{L^{2}\left(L^{2}\right)}=\left\{\sum_{n} \sum_{\ell}\left(\frac{e_{\ell}^{n+1}-e_{\ell}^{n}}{k}\right)^{2} h^{2} k\right\}^{1 / 2},
$$

and, for the vector solution $\mathbf{q}$,

$$
\||\sigma|\|_{L^{\infty}\left(L^{2}\right)}=\max _{n}\left\{\sum_{i}\left(\sigma_{x, i}^{n}\right)^{2} h^{2}+\sum_{j}\left(\sigma_{y, j}^{n}\right)^{2} h^{2}\right\}^{1 / 2},
$$

where $\sigma^{n}=\mathbf{q}^{n}-\mathbf{q}_{h}^{n}$ are the errors at the center of the cell edges in the $x$ and $y$ directions, respectively, which are $\mathcal{O}\left(h^{2}\right)$ close to $\pi \sigma$. Again, these norms should exhibit superconvergence of order $\mathcal{O}\left(h^{2}+k^{2}\right)$.

Note that we should use a scaling of $h \sim k$, since the overall error is $\mathcal{O}\left(h^{2}+k^{2}\right)$. However, the condition $k / h^{2} \rightarrow 0$ is required for the theoretical results. We have been unable to 


\begin{tabular}{c|c|c|c|c|c|c} 
Error & Method & $n=20$ & $n=40$ & $n=80$ & $n=160$ & Rate \\
\hline \hline$\|\mid\| \|_{L^{\infty}\left(L^{2}\right)}$ & C-N & $6.51 \mathrm{e}-3$ & $1.51 \mathrm{e}-3$ & $3.79 \mathrm{e}-4$ & $9.47 \mathrm{e}-5$ & 2.03 \\
& AD & $6.05 \mathrm{e}-3$ & $1.51 \mathrm{e}-3$ & $3.79 \mathrm{e}-4$ & $9.47 \mathrm{e}-5$ & 2.00 \\
& AD-M & $6.09 \mathrm{e}-3$ & $1.51 \mathrm{e}-3$ & $3.79 \mathrm{e}-4$ & $9.47 \mathrm{e}-5$ & 2.00 \\
\hline$\left\|e_{t}\right\|_{L^{2}\left(L^{2}\right)}$ & C-N & $2.21 \mathrm{e}-2$ & $6.18 \mathrm{e}-3$ & $1.68 \mathrm{e}-3$ & $4.46 \mathrm{e}-4$ & 1.88 \\
& AD & $1.90 \mathrm{e}-2$ & $5.95 \mathrm{e}-3$ & $1.68 \mathrm{e}-3$ & $4.49 \mathrm{e}-4$ & 1.80 \\
& AD-M & $1.10 \mathrm{e}-2$ & $3.28 \mathrm{e}-3$ & $1.15 \mathrm{e}-3$ & $3.66 \mathrm{e}-4$ & 1.62 \\
\hline$\|\sigma \mid\|_{L^{\infty}\left(L^{2}\right)}$ & C-N & $6.83 \mathrm{e}-2$ & $1.77 \mathrm{e}-2$ & $4.58 \mathrm{e}-3$ & $1.17 \mathrm{e}-3$ & 1.95 \\
& AD & $4.89 \mathrm{e}-2$ & $1.43 \mathrm{e}-2$ & $4.27 \mathrm{e}-3$ & $1.17 \mathrm{e}-3$ & 1.79 \\
& AD-M & $8.44 \mathrm{e}-2$ & $2.08 \mathrm{e}-2$ & $4.91 \mathrm{e}-3$ & $1.18 \mathrm{e}-3$ & 2.06
\end{tabular}

Table 1: Discrete errors with exact solution $u_{+}$, and the observed convergence rates.

find an example that requires this condition, however. Thus, we use a single discretization parameter $n$ so that $h=k=1 / n$. We note in passing that the condition $k / h^{2} \rightarrow 0$ is natural for the Backward Euler methods combined with $R T_{0}$, since then the superconvergent errors are $\mathcal{O}\left(h^{2}+k\right)$.

In our AD-M method, for practical purposes, $\lambda^{1}$ was obtained by running 10 micro-time steps of AD using one-tenth of the time step. We use the unit square as $\Omega$ and $T=1.0$. In all our test cases, we choose a specific solution $u(x, y, t)$ and coefficient $a(x, y)$, and then we determine $f, g$, and $u_{0}$ so that (4.2)-(4.5) are satisfied.

\subsection{Smooth examples.}

In this set of examples, based on those of [7]. In Table 1, we show the results for the exact solution

$$
u_{+}(x, y, t)=\sin (2 \pi t)+\sin (2 \pi x)+\sin (2 \pi y)
$$

and $a(x, y)=1$, for which $f(x, y)=2 \cos (2 \pi t) \pi+4 \sin (2 \pi x) \pi^{2}+4 \sin (2 \pi y) \pi^{2}$. In this example, the $\mathrm{AD}$ method does not introduce a larger splitting error, so all three methods are comparable in their errors, at least for large values of $n$ (i.e., small values of $h=k$ ). We see second-order convergence for the full Crank-Nicolson system for all three norms. Moreover, we see nearly second-order convergence for the two alternating direction methods, but the rate is somewhat degraded to about 1.6 to 1.8 for some of the norms. It appears that in this simple example, the splitting errors actually cancel some of the approximation error, giving less overall error for $\mathrm{AD}$ and $\mathrm{AD}-\mathrm{M}$ than for $\mathrm{C}-\mathrm{N}$ in some norms for small values of $n$.

In Table 2, we show the results for the exact solution

$$
u_{\times}(x, y, t)=(\sin (2 \pi t)+1)(\sin (2 \pi x)+1)(\sin (2 \pi y)+1)
$$

and diffusion coefficient $a(x, y)=1$. We see second-order convergence for $\mathrm{C}-\mathrm{N}$. In this example, the AD method has much more error than C-N. AD produces a large splitting error that degrades the effectiveness of the algorithm. It does not even appear that we have entered the region of asymptotic convergence for this method, since the convergence rates are less than expected for $e_{t}$ and $\sigma$.

On the other hand, the AD-M method produces an error larger but comparable to C-N. The AD-M splitting error is much smaller than that for AD. We observe somewhat better 


\begin{tabular}{c|c|c|c|c|c|c} 
Error & Method & $n=20$ & $n=40$ & $n=80$ & $n=160$ & Rate \\
\hline \hline$\|\mid\|_{L^{\infty}\left(L^{2}\right)}$ & C-N & $1.09 \mathrm{e}-2$ & $2.73 \mathrm{e}-3$ & $6.81 \mathrm{e}-4$ & $1.70 \mathrm{e}-4$ & 2.00 \\
& AD & $6.28 \mathrm{e}-2$ & $1.58 \mathrm{e}-2$ & $3.94 \mathrm{e}-3$ & $9.86 \mathrm{e}-4$ & 2.00 \\
& AD-M & $2.70 \mathrm{e}-2$ & $4.50 \mathrm{e}-3$ & $8.84 \mathrm{e}-4$ & $1.94 \mathrm{e}-4$ & 2.37 \\
\hline\|\|$_{t}\|\|_{L^{2}\left(L^{2}\right)}$ & C-N & $2.86 \mathrm{e}-2$ & $7.84 \mathrm{e}-3$ & $2.12 \mathrm{e}-3$ & $5.63 \mathrm{e}-4$ & 1.89 \\
& AD & $2.59 \mathrm{e}-1$ & $6.92 \mathrm{e}-2$ & $2.27 \mathrm{e}-2$ & $8.63 \mathrm{e}-3$ & 1.63 \\
& AD-M & $9.75 \mathrm{e}-2$ & $1.33 \mathrm{e}-2$ & $2.19 \mathrm{e}-3$ & $5.10 \mathrm{e}-4$ & 2.53 \\
\hline$\|\mid\|_{L^{\infty}\left(L^{2}\right)}$ & C-N & $1.67 \mathrm{e}-1$ & $4.15 \mathrm{e}-2$ & $1.03 \mathrm{e}-2$ & $2.59 \mathrm{e}-3$ & 2.00 \\
& AD & $1.80 \mathrm{e}-0$ & $6.45 \mathrm{e}-1$ & $2.70 \mathrm{e}-1$ & $1.17 \mathrm{e}-1$ & 1.31 \\
& AD-M & $6.40 \mathrm{e}-1$ & $1.14 \mathrm{e}-1$ & $2.14 \mathrm{e}-2$ & $4.20 \mathrm{e}-3$ & 2.42
\end{tabular}

Table 2: Discrete errors with exact solution $u_{\times}$, and the observed convergence rate.

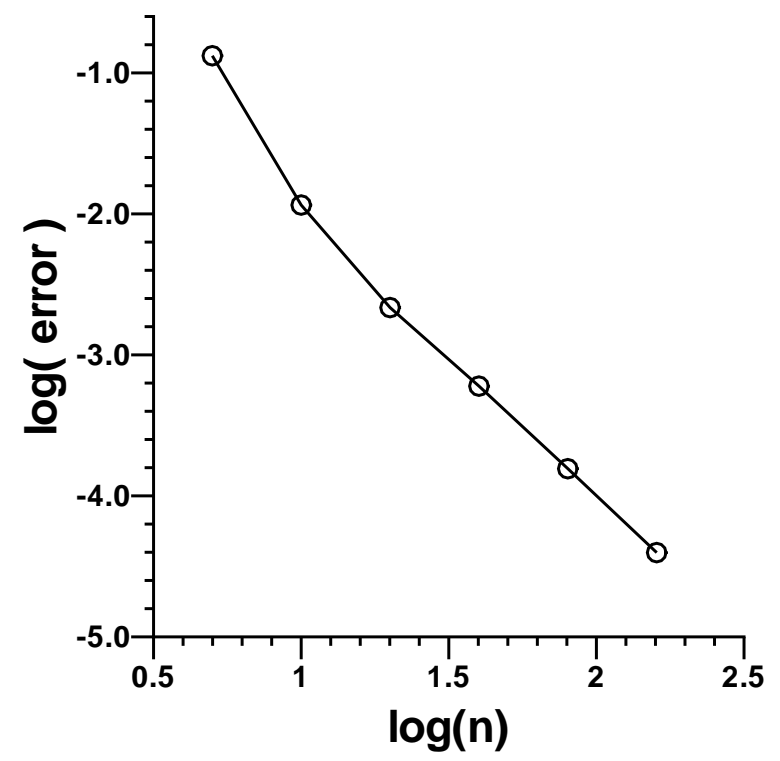

Figure 1: The log of the error $\|\mid e\|_{L^{\infty}\left(L^{2}\right)}$ versus the $\log$ of $n$.

rates of convergence (greater than 2), because the splitting error is being removed at the rate of $\mathcal{O}\left(k^{3}\right)$.

We show the reduction in splitting error in Fig. 1, where we plot the base $10 \log$ of the error \|\|$e \|_{L^{\infty}\left(L^{2}\right)}$ for the base $10 \log$ of $n$, where $n=5,10,20,40,80$, and 160. The data are for exact solution $u(x, y, t)=(\sin (\pi t)+1)(\sin (\pi x)+1)(\sin (\pi y)+1)$. The graph clearly shows a slope of about 3 , i.e., $\mathcal{O}\left(k^{3}\right)$ convergence, for small $n$. The slope quickly reduces to about 2, i.e., $\mathcal{O}\left(k^{2}\right)$ convergence.

In our final smooth experiment, we test our $\mathrm{AD}-\mathrm{M}$ with a variable coefficient. We again 


\begin{tabular}{c|c|c|c|c} 
Error & Method & $a=a_{1}$ & $a=a_{2}$ & $a=a_{3}$ \\
\hline \hline$\|\mid\| \|_{L^{\infty}\left(L^{2}\right)}$ & C-N & $6.81 \mathrm{e}-4$ & $6.79 \mathrm{e}-4$ & $6.85 \mathrm{e}-4$ \\
& AD & $3.94 \mathrm{e}-3$ & $2.43 \mathrm{e}-3$ & $6.08 \mathrm{e}-3$ \\
& AD-M & $8.84 \mathrm{e}-4$ & $7.77 \mathrm{e}-4$ & $9.64 \mathrm{e}-4$ \\
\hline$\left\|e_{t}\right\|_{L^{2}\left(L^{2}\right)}$ & C-N & $2.12 \mathrm{e}-3$ & $1.87 \mathrm{e}-3$ & $3.59 \mathrm{e}-3$ \\
& AD & $2.27 \mathrm{e}-2$ & $1.49 \mathrm{e}-2$ & $3.00 \mathrm{e}-2$ \\
& AD-M & $2.19 \mathrm{e}-3$ & $1.91 \mathrm{e}-3$ & $3.24 \mathrm{e}-3$ \\
\hline$\|\sigma \mid\|_{L^{\infty}\left(L^{2}\right)}$ & C-N & $1.03 \mathrm{e}-2$ & $6.40 \mathrm{e}-3$ & $1.74 \mathrm{e}-2$ \\
& AD & $2.70 \mathrm{e}-1$ & $1.37 \mathrm{e}-1$ & $5.29 \mathrm{e}-1$ \\
& AD-M & $2.14 \mathrm{e}-2$ & $1.17 \mathrm{e}-2$ & $4.49 \mathrm{e}-2$
\end{tabular}

Table 3: Discrete $L^{2}$-errors for $n=80$ with exact solution $u_{\times}$.

take the exact solution $u_{\times}$, but set $a$ to one of the three choices

$$
\begin{aligned}
& a_{1}(x, y)=1, \\
& a_{2}(x, y)=\frac{1}{2+\cos (3 \pi x) \cos (2 \pi y)}, \\
& a_{3}(x, y)= \begin{cases}1+0.5 \sin (5 \pi x)+y^{3}, & \text { if } \quad x \leq 0.5, \\
\frac{1.5}{1+(x-0.5)^{2}}+y^{3}, & \text { otherwise. }\end{cases}
\end{aligned}
$$

In Table 3, we present the results, which show again that $\mathrm{AD}$ is inferior to $\mathrm{AD}-\mathrm{M}$, which is comparable to the full solution.

\subsection{Nonsmooth examples.}

In this set of examples, we consider the true solution

$$
u_{\alpha}(x, y, t)=|x y t-0.25|^{\alpha},
$$

for some parameter $\alpha$. Note that the solution has bounded partial derivatives (of any type) only up to order $\alpha$. According to Theorem 6.1, we should take $\alpha>3$ to have the regularity demanded of the solution for superconvergence, and $\alpha>2$ to obtain convergence of order $\mathcal{O}(k+h)$ (since the time error will degenerate to first order).

In the first set of tests, we take $a=1$, which means that the 1 -D operators commute. It is reasonable to expect that there is no requirement that $k / h^{2}$ be sufficiently small in this case, assuming the results of Douglas and Kim [7] extend to mixed methods. Indeed we see in Table 4 good convergence for this case when $\alpha=3.1$, meaning that $\mathbf{q}$ has 2 derivatives.

When $\alpha=2.1$, C-N behaves as expected in Table 5, which shows the convergence rate of $e_{t}$ and $\sigma$ degrading to about $\mathcal{O}(k+h)$. The fact that the error norm of $e$ retains its superconvergence is unexpected. Both $\mathrm{AD}$ and $\mathrm{AD}-\mathrm{M}$ follow the general results of $\mathrm{C}-\mathrm{N}$, but with somewhat greater error (and $\mathrm{AD}$ is worse that $\mathrm{AD}-\mathrm{M}$ ). When $\alpha=1.9$ (Table 6 ), we lose sufficient regularity to have full good convergence, but we nevertheless retain a fractional rate of convergence (as we should expect) of order about 0.9 for $e_{t}$ and $\sigma$, and a bit better partial superconvergence rate for $e$. 


\begin{tabular}{c|c|c|c|c|c|c} 
Error & Method & $n=20$ & $n=40$ & $n=80$ & $n=160$ & Rate \\
\hline \hline$\|\mid\|_{L^{\infty}\left(L^{2}\right)}$ & C-N & $1.14 \mathrm{e}-4$ & $2.85 \mathrm{e}-5$ & $7.13 \mathrm{e}-6$ & $1.78 \mathrm{e}-6$ & 2.00 \\
& AD & $1.32 \mathrm{e}-3$ & $3.41 \mathrm{e}-4$ & $8.62 \mathrm{e}-5$ & $2.16 \mathrm{e}-5$ & 2.00 \\
& AD-M & $2.52 \mathrm{e}-4$ & $4.51 \mathrm{e}-5$ & $9.10 \mathrm{e}-6$ & $2.02 \mathrm{e}-6$ & 2.32 \\
\hline\|\|$_{t} \|_{L^{2}\left(L^{2}\right)}$ & C-N & $1.84 \mathrm{e}-4$ & $4.63 \mathrm{e}-5$ & $1.18 \mathrm{e}-5$ & $2.97 \mathrm{e}-6$ & 1.98 \\
& AD & $1.78 \mathrm{e}-3$ & $4.63 \mathrm{e}-4$ & $1.18 \mathrm{e}-4$ & $3.00 \mathrm{e}-5$ & 1.97 \\
& AD-M & $5.28 \mathrm{e}-4$ & $1.23 \mathrm{e}-4$ & $3.12 \mathrm{e}-5$ & $8.23 \mathrm{e}-6$ & 2.00 \\
\hline$\|\mid\| \|_{L^{\infty}\left(L^{2}\right)}$ & C-N & $8.71 \mathrm{e}-4$ & $2.17 \mathrm{e}-4$ & $5.49 \mathrm{e}-5$ & $1.38 \mathrm{e}-5$ & 1.99 \\
& AD & $5.29 \mathrm{e}-2$ & $2.02 \mathrm{e}-2$ & $7.42 \mathrm{e}-3$ & $2.67 \mathrm{e}-3$ & 1.44 \\
& AD-M & $6.96 \mathrm{e}-3$ & $1.36 \mathrm{e}-3$ & $2.49 \mathrm{e}-4$ & $4.79 \mathrm{e}-5$ & 2.40
\end{tabular}

Table 4: Discrete errors with exact solution $u_{3.1}$ and $a=1$, and the observed convergence rates.

\begin{tabular}{c|c|c|c|c|c|c} 
Error & Method & $n=20$ & $n=40$ & $n=80$ & $n=160$ & Rate \\
\hline \hline$\|\mid\| \|_{L^{\infty}\left(L^{2}\right)}$ & C-N & $1.04 \mathrm{e}-4$ & $2.65 \mathrm{e}-5$ & $9.06 \mathrm{e}-6$ & $1.89 \mathrm{e}-6$ & 1.89 \\
& AD & $8.78 \mathrm{e}-4$ & $2.22 \mathrm{e}-4$ & $5.75 \mathrm{e}-5$ & $1.38 \mathrm{e}-5$ & 1.99 \\
& AD-M & $1.39 \mathrm{e}-4$ & $3.02 \mathrm{e}-5$ & $9.43 \mathrm{e}-6$ & $2.09 \mathrm{e}-6$ & 1.99 \\
\hline$\left\|e_{t}\right\|_{L^{2}\left(L^{2}\right)}$ & C-N & $6.90 \mathrm{e}-4$ & $4.41 \mathrm{e}-4$ & $2.37 \mathrm{e}-4$ & $1.08 \mathrm{e}-4$ & 0.89 \\
& AD & $1.02 \mathrm{e}-3$ & $3.78 \mathrm{e}-4$ & $1.66 \mathrm{e}-4$ & $7.10 \mathrm{e}-5$ & 1.27 \\
& AD-M & $7.51 \mathrm{e}-4$ & $3.70 \mathrm{e}-4$ & $1.85 \mathrm{e}-4$ & $8.47 \mathrm{e}-5$ & 1.04 \\
\hline$\|\sigma\|_{L^{\infty}\left(L^{2}\right)}$ & C-N & $2.04 \mathrm{e}-3$ & $9.24 \mathrm{e}-4$ & $4.90 \mathrm{e}-4$ & $2.07 \mathrm{e}-4$ & 1.08 \\
& AD & $2.94 \mathrm{e}-2$ & $1.07 \mathrm{e}-2$ & $3.87 \mathrm{e}-3$ & $1.39 \mathrm{e}-3$ & 1.47 \\
& AD-M & $2.93 \mathrm{e}-3$ & $9.24 \mathrm{e}-4$ & $3.75 \mathrm{e}-4$ & $1.62 \mathrm{e}-4$ & 1.38
\end{tabular}

Table 5: Discrete errors with exact solution $u_{2.1}$ and $a=1$, and the observed convergence rates.

\begin{tabular}{c|c|c|c|c|c|c} 
Error & Method & $n=20$ & $n=40$ & $n=80$ & $n=160$ & Rate \\
\hline \hline\|\|$e \|_{L^{\infty}\left(L^{2}\right)}$ & C-N & $1.74 \mathrm{e}-4$ & $5.07 \mathrm{e}-5$ & $1.73 \mathrm{e}-5$ & $5.30 \mathrm{e}-6$ & 1.67 \\
& AD & $8.49 \mathrm{e}-4$ & $2.15 \mathrm{e}-4$ & $5.17 \mathrm{e}-5$ & $1.49 \mathrm{e}-5$ & 1.96 \\
& AD-M & $1.84 \mathrm{e}-4$ & $5.33 \mathrm{e}-5$ & $1.48 \mathrm{e}-5$ & $4.98 \mathrm{e}-6$ & 1.75 \\
\hline\|\|$e_{t}\|\|_{L^{2}\left(L^{2}\right)}$ & C-N & $1.64 \mathrm{e}-3$ & $1.47 \mathrm{e}-3$ & $9.17 \mathrm{e}-4$ & $4.43 \mathrm{e}-4$ & 0.66 \\
& AD & $1.37 \mathrm{e}-3$ & $9.13 \mathrm{e}-4$ & $5.50 \mathrm{e}-4$ & $2.56 \mathrm{e}-4$ & 0.80 \\
& AD-M & $1.36 \mathrm{e}-3$ & $9.48 \mathrm{e}-4$ & $5.80 \mathrm{e}-4$ & $2.77 \mathrm{e}-4$ & 0.76 \\
\hline$\|\mid \sigma\|_{L^{\infty}\left(L^{2}\right)}$ & C-N & $4.55 \mathrm{e}-3$ & $2.66 \mathrm{e}-3$ & $1.68 \mathrm{e}-3$ & $7.71 \mathrm{e}-4$ & 0.83 \\
& AD & $2.67 \mathrm{e}-2$ & $9.79 \mathrm{e}-3$ & $3.55 \mathrm{e}-3$ & $1.29 \mathrm{e}-3$ & 1.46 \\
& AD-M & $3.73 \mathrm{e}-3$ & $1.63 \mathrm{e}-3$ & $8.19 \mathrm{e}-4$ & $3.84 \mathrm{e}-4$ & 1.08
\end{tabular}

Table 6: Discrete errors with exact solution $u_{1.9}$ and $a=1$, and the observed convergence rates.

In the second set of nonsmooth tests, we also take a nonsmooth $a$ given by

$$
a(x, y)= \begin{cases}2+\sin \left(x y^{2}\right)+32(x-0.5)(y-0.5), & x \leq 0.5, y \leq 0.5 \\ 2+\sin \left(x y^{2}\right)+8(x-0.5)(y-0.5), & x>0.5, y>0.5 \\ 2+\sin \left(x y^{2}\right), & \text { otherwise }\end{cases}
$$




\begin{tabular}{c|c|c|c|c|c|c} 
Error & Method & $n=20$ & $n=40$ & $n=80$ & $n=160$ & Rate \\
\hline \hline$\|\mid\|_{L^{\infty}\left(L^{2}\right)}$ & C-N & $7.04 \mathrm{e}-5$ & $1.77 \mathrm{e}-5$ & $4.44 \mathrm{e}-6$ & $1.11 \mathrm{e}-6$ & 2.00 \\
& AD & $5.11 \mathrm{e}-3$ & $1.47 \mathrm{e}-3$ & $3.88 \mathrm{e}-4$ & $9.88 \mathrm{e}-5$ & 1.90 \\
& AD-M & $8.32 \mathrm{e}-4$ & $1.11 \mathrm{e}-4$ & $1.56 \mathrm{e}-5$ & $2.41 \mathrm{e}-6$ & 2.81 \\
\hline\|\|$_{t}\|\|_{L^{2}\left(L^{2}\right)}$ & C-N & $1.91 \mathrm{e}-4$ & $4.40 \mathrm{e}-5$ & $1.12 \mathrm{e}-5$ & $2.93 \mathrm{e}-6$ & 2.01 \\
& AD & $6.97 \mathrm{e}-3$ & $1.97 \mathrm{e}-3$ & $5.17 \mathrm{e}-4$ & $1.33 \mathrm{e}-4$ & 1.91 \\
& AD-M & $1.57 \mathrm{e}-3$ & $3.27 \mathrm{e}-4$ & $7.77 \mathrm{e}-5$ & $1.99 \mathrm{e}-5$ & 2.10 \\
\hline\|\|$\sigma \|_{L^{\infty}\left(L^{2}\right)}$ & C-N & $5.03 \mathrm{e}-3$ & $1.26 \mathrm{e}-3$ & $3.16 \mathrm{e}-4$ & $7.92 \mathrm{e}-5$ & 2.00 \\
& AD & $7.89 \mathrm{e}-1$ & $3.70 \mathrm{e}-1$ & $1.51 \mathrm{e}-1$ & $5.76 \mathrm{e}-2$ & 1.26 \\
& AD-M & $1.46 \mathrm{e}-1$ & $2.87 \mathrm{e}-2$ & $5.23 \mathrm{e}-3$ & $9.46 \mathrm{e}-4$ & 2.43
\end{tabular}

Table 7: Discrete errors with exact solution $u_{3.1}$ and nonsmooth $a$, and the observed convergence rates.

\begin{tabular}{c|c|c|c|c|c|c} 
Error & Method & $n=20$ & $n=40$ & $n=80$ & $n=160$ & Rate \\
\hline \hline$\|\mid\|_{L^{\infty}\left(L^{2}\right)}$ & C-N & $7.14 \mathrm{e}-5$ & $2.34 \mathrm{e}-5$ & $8.24 \mathrm{e}-6$ & $1.91 \mathrm{e}-6$ & 1.72 \\
& AD & $3.46 \mathrm{e}-3$ & $9.15 \mathrm{e}-4$ & $2.34 \mathrm{e}-4$ & $5.85 \mathrm{e}-5$ & 1.96 \\
& AD-M & $3.37 \mathrm{e}-4$ & $5.61 \mathrm{e}-5$ & $1.18 \mathrm{e}-5$ & $3.09 \mathrm{e}-6$ & 2.26 \\
\hline$\left\|\left.\right|_{t}\right\| \|_{L^{2}\left(L^{2}\right)}$ & C-N & $9.14 \mathrm{e}-4$ & $6.44 \mathrm{e}-4$ & $3.49 \mathrm{e}-4$ & $1.60 \mathrm{e}-4$ & 0.84 \\
& AD & $3.90 \mathrm{e}-3$ & $1.12 \mathrm{e}-3$ & $3.67 \mathrm{e}-4$ & $1.37 \mathrm{e}-4$ & 1.61 \\
& AD-M & $1.59 \mathrm{e}-3$ & $6.51 \mathrm{e}-4$ & $3.09 \mathrm{e}-4$ & $1.42 \mathrm{e}-4$ & 1.15 \\
\hline$\|\sigma\|_{L^{\infty}\left(L^{2}\right)}$ & C-N & $5.58 \mathrm{e}-3$ & $2.41 \mathrm{e}-3$ & $1.24 \mathrm{e}-3$ & $5.28 \mathrm{e}-4$ & 1.12 \\
& AD & $4.61 \mathrm{e}-1$ & $1.92 \mathrm{e}-1$ & $7.37 \mathrm{e}-2$ & $2.71 \mathrm{e}-2$ & 1.36 \\
& AD-M & $4.64 \mathrm{e}-2$ & $1.32 \mathrm{e}-2$ & $4.47 \mathrm{e}-3$ & $1.71 \mathrm{e}-3$ & 1.59
\end{tabular}

Table 8: Discrete errors with exact solution $u_{2.1}$ and nonsmooth $a$, and the observed convergence rates.

\begin{tabular}{c|c|c|c|c|c|c} 
Error & Method & $n=20$ & $n=40$ & $n=80$ & $n=160$ & Rate \\
\hline \hline$\|\mid\|_{L^{\infty}\left(L^{2}\right)}$ & C-N & $1.85 \mathrm{e}-4$ & $6.77 \mathrm{e}-5$ & $2.50 \mathrm{e}-5$ & $6.64 \mathrm{e}-6$ & 1.58 \\
& AD & $3.08 \mathrm{e}-3$ & $8.02 \mathrm{e}-4$ & $1.97 \mathrm{e}-4$ & $5.21 \mathrm{e}-5$ & 1.97 \\
& AD-M & $3.68 \mathrm{e}-4$ & $7.70 \mathrm{e}-5$ & $2.35 \mathrm{e}-5$ & $6.84 \mathrm{e}-6$ & 1.90 \\
\hline\|\|$e_{t} \|_{L^{2}\left(L^{2}\right)}$ & C-N & $2.15 \mathrm{e}-3$ & $2.20 \mathrm{e}-3$ & $1.38 \mathrm{e}-3$ & $6.66 \mathrm{e}-4$ & 0.86 \\
& AD & $3.77 \mathrm{e}-3$ & $1.55 \mathrm{e}-3$ & $8.30 \mathrm{e}-4$ & $3.89 \mathrm{e}-4$ & 1.07 \\
& AD-M & $2.41 \mathrm{e}-3$ & $1.42 \mathrm{e}-3$ & $8.64 \mathrm{e}-4$ & $4.24 \mathrm{e}-4$ & 0.82 \\
\hline\|\|$\sigma \|_{L^{\infty}\left(L^{2}\right)}$ & C-N & $1.15 \mathrm{e}-2$ & $6.72 \mathrm{e}-3$ & $4.17 \mathrm{e}-3$ & $1.93 \mathrm{e}-3$ & 0.84 \\
& AD & $3.86 \mathrm{e}-1$ & $1.57 \mathrm{e}-1$ & $5.97 \mathrm{e}-2$ & $2.19 \mathrm{e}-2$ & 1.38 \\
& AD-M & $5.41 \mathrm{e}-2$ & $1.49 \mathrm{e}-2$ & $7.24 \mathrm{e}-3$ & $3.60 \mathrm{e}-3$ & 1.03
\end{tabular}

Table 9: Discrete errors with exact solution $u_{1.9}$ and nonsmooth $a$, and the observed convergence rates.

In this case, the 1-D operators do not commute. Nevertheless, we do not seem to require that $k / h^{2}$ be sufficiently small (contrary to what Theorem 6.1 suggests). The results for $\alpha=3.1$, 2.1, and 1.9 are given in Tables $7-9$, and they agree qualitatively with the previous test cases. 


\section{Conclusions.}

We have shown that the $\mathrm{AD}$ and $\mathrm{AD}-\mathrm{M}$ algorithms for finite difference and Galerkin approximations to second order parabolic equations converge optimally if only $k / h^{2} \rightarrow 0$ (not $\left.k / h^{4} \rightarrow 0\right)$.

We have shown that the AD-M modification in [7] can be applied to mixed finite element procedures. Moreover, we formulated an efficient Uzawa AD-M implementation. The resulting method has splitting error of size $\mathcal{O}\left(k^{3}\right)$. For $R T_{s}$, the Uzawa AD and AD-M converge optimally at the rate $\mathcal{O}\left(k^{r}+h^{s+1}\right.$ provided that $k / h^{2} \rightarrow 0$, where $r=1$ for backward Euler and $r=2$ for Crank-Nicolson time discretization. Moreover, both methods exhibit superconvergence. In discrete norms, the scalar and vector variables converge with to order $\mathcal{O}\left(k^{r}+h^{s+2}\right.$.

Numerical results using Crank-Nicolson and $R T_{0}$ show that $k \sim h$ works well for AD and AD-M, suggesting that the condition $k / h^{2} \rightarrow 0$ is not actually needed (though we cannot now prove this). The numerical results also clearly show that the splitting error is higher order, and was seen to be $\mathcal{O}\left(k^{3}\right)$. Generally, we saw that the AD-M error was comparable to $\mathrm{C}-\mathrm{N}$, but AD had more error.

It is clear that from the algorithms that th $\mathrm{AD}-\mathrm{M}$ modification requires little extra computation compared to $\mathrm{AD}$, but it can lead to a significant reduction in the splitting perturbation error associated with the $\mathrm{AD}$ method for mixed finite elements. Moreover, AD-M is much easier to solve than $\mathrm{C}-\mathrm{N}$ alone, but often produces comparable error.

\section{References}

[1] S. C. Brenner and L. R. Scott, The Mathematical Theory of Finite Element Methods, Springer-Verlag, New York, 1994.

[2] F. Brezzi, J. Douglas, Jr., M. Fortin, and L. D. Marini, Efficient rectangular mixed finite elements in two and three space variables, RAIRO Modél. Math. Anal. Numér., 21, (1987), 581-604.

[3] F. Brezzi and M. Fortin, Mixed and Hybrid Finite Element Methods, Springer-Verlag, New York, 1991.

[4] J. Douglas, Jr., On the numerical integration of $\frac{\partial^{2} u}{\partial x^{2}}+\frac{\partial^{2} u}{\partial y^{2}}=\frac{\partial u}{\partial t}$ by implicit methods, J. Soc. Indust. Appl. Math., 3 (1955), 42-65.

[5] J. Douglas, Jr., and T. Dupont, Alternating-direction Galerkin methods on rectangles, in Numerical Solution of Partial Differential Equations, II (SYNSPADE 1970), Academic Press, 1971, pp. 133-214.

[6] J. Douglas, Jr., and J. Gunn, A general formulation of alternating direction methods: Part I. Parabolic and hyperbolic problems, Numer. Math., 6 (1964), pp. 428-453.

[7] J. Douglas, Jr., and S. Kim, Improved accuracy for locally one-dimensional methods for parabolic equations, Math. Models and Methods in Appl. Sci., 11 (2001), 1563-1579.

[8] J. Douglas, Jr., and D. W. Peaceman, Numerical solution of two-dimensional heat flow problems, Amer. Inst. of Chem. Engng. J., 1 (1955), 505-512. 
[9] J. Douglas, Jr., and P. Pietra, A description of some alternating-direction iterative techniques for mixed finite element methods, in "Mathematical and Computational Methods in Seismic Exploration and Reservoir Modeling," W. E. Fitzgibbon (ed.), SIAM, Philadelphia, PA, 1986, pp. 37-53.

[10] J. Douglas, Jr., and Junping Wang, Superconvergence for mixed finite element methods on rectangular domains, Calcolo, 26 (1989), 121-134.

[11] R. Durán, Superconvergence for rectangular mixed finite elements, Numer. Math., 58 (1990), 287-298.

[12] M. Nakata, A. Weiser, and M. F. Wheeler, Some superconvergence results for mixed finite element methods for elliptic problems on rectangular domains, in The Mathematics of Finite Elements and Applications V, J. R. Whiteman (ed.), Academic Press, London, 1985 .

[13] D. W. Peaceman and H. Rachford, The numerical solution of parabolic and elliptic equations, J. Soc. Indust. Appl. Math., 3 (1955), 28-41.

[14] R. A. Raviart and J. M. Thomas, A mixed finite element method for 2nd order elliptic problems, in "Mathematical Aspects of Finite Element Methods," Lecture Notes in Math. 606, I. Galligani and E. Magenes (eds.), Springer-Verlag, New York, 1977, pp. 292-315.

[15] M. F. Wheeler, A priori $L_{2}$ error estimates for Galerkin approximations to parabolic partial differential equations, SIAM J. Numer. Anal., 10 (1973), 723-759. 